\title{
Impaired primordial follicle assembly in offspring ovaries from zearalenone exposed mothers involves reduced mitochondrial activity and altered epigenetics in oocytes
}

\author{
Yan-Qin Feng \\ Qingdao Agricultural University \\ Jun-Jie Wang \\ Qingdao Agricultural University \\ Ming-Hao Li \\ Qingdao Agricultural University \\ Yu Tian \\ Qingdao Agricultural University
}

Ai-Hong Zhao

Qingdao academy of agricultural sciencess

Lan Li

Qingdao Agricultural University

Massimo De Felici

University of Rome Tor Vergata Department of Biomedicine and Prevention: Universita degli Studi di Roma Tor Vergata Dipartimento di Biomedicina e Prevenzione

Wei Shen ( $\boldsymbol{\sigma}$ wshen@qau.edu.cn )

Qingdao Agricultural University https://orcid.org/0000-0001-6112-2501

\section{Research Article}

Keywords: zearalenone, primordial follicle assembly, mitochondrial activity, single-cell transcriptome, histone deacetylase 2

Posted Date: February 7th, 2022

DOI: https://doi.org/10.21203/rs.3.rs-1306300/v1

License: (c) (1) This work is licensed under a Creative Commons Attribution 4.0 International License. Read Full License 
Version of Record: A version of this preprint was published at Cellular and Molecular Life Sciences on April 26th, 2022. See the published version at https://doi.org/10.1007/s00018-022-04288-0. 


\section{Abstract}

Previous works have shown that zearalenone (ZEA), as an estrogenic pollutant, has adverse effects on mammalian folliculogenesis. In the present study, we found that prolonged exposure of female mice to ZEA around the end of pregnancy, caused severe impairment of primordial follicle formation in the ovaries of newborn mice and altered the expression of many genes in oocytes as revealed by scRNA-seq. These changes were associated with morphological and molecular alterations of mitochondria, increased autophagic markers in oocytes, and epigenetic changes in the ovaries of newborn mice from ZEA exposed mothers. The latter increased expression of HDAC2 deacetylases was leading to decreased levels of H3K9ac and H4K12ac. Most of these modifications were relieved when the expression of HDAC2 in newborn ovaries was reduced by RNA interference during in vitro culture in the presence of ZEA. Such changes were also alleviated in offspring ovaries from mothers treated with both ZEA and the ubiquinone coenzyme Q10 (CoQ10), which is known to be able to restore mitochondrial activities. We concluded that impaired mitochondrial activities in oocytes caused by ZEA are at the origin of metabolic alterations that modify the expression of genes controlling autophagy and primordial follicle assembly through changes in epigenetic histones.

\section{Introduction}

In mammals, the primordial follicle (PF) pool within the ovaries is established during the perinatal period and constitutes the "ovarian reserve" (OR), largely determining female reproductive lifespan $[1,2]$. The assembly of PFs requires individual oocytes to associate with pregranulosa cells, which involves a complex process of breakdown of germ cell cysts and parallel recruitment of pregranulosa cells [3-5]. After PF formation, fine mechanisms ensure that the majority of PFs are held quiescent and remain poised to participate in follicle growth for long periods which last for decades in humans. Some evidence exists that throughout this extended period, various compounds including environmental toxicants as endocrine disruptors, heavy metals, pesticides, tobacco smoke, chemicals used in plastic, and cosmetic and sanitary products, can adversely affect the OR and consequently impair female fertility [6-10].

Many studies have been performed on zearalenone (ZEA), a mycotoxin produced by Fusarium and Gibberella species, the world-wide occurrence of which has been reported in foodstuffs [11-13]. These studies have shown that ZEA exposure may cause serious hepatotoxicity and reproductive toxicity [14$16]$. Owing to the similarity of its chemical structure with endogenous estrogen ( $17 \beta$-estradiol; $E_{2}$ ), the toxicity of ZEA on the female reproductive system has received widespread attention [17-20]. Relevant to the present work, some papers show that the drugs can impair PF formation [21-23]. Other researches have reported that $\mathrm{PF}$ formation can be inhibited by exposure to environmental estrogens and high concentrations of $E_{2}$, and ZEA exposure significantly inhibit PF formation in embryonic stage E17.5 or postnatal 0 day (PD0) mouse ovaries [24, 25]. The adverse effects of ZEA on PF assembly have been explained on the basis of its estrogenic action. 
The toxicity of ZEA and its metabolites can be caused through other mechanisms such as oxidative stress and epigenetic changes [26, 27]. In fact, it has been reported that ZEA can increase reactive oxygen species (ROS) level, causing DNA damage and consequent apoptosis in many cell types [28, 29]. In this regard, Liu et al. reported that maternal exposure to ZEA increased DNA-DSBs in the ovaries of mouse fetuses and this was associated with a remarkable reduction of the number of PFs in the ovaries of newborn pups [30]. In line with this, Kong et al. found that impairment of PF assembly in the ovaries of suckled mouse offspring of ZEA exposure mothers was associated with a significant reduction of the levels of PF assembly specific oocyte genes such as Figla, Lhx8, Sohlh2, and Nobox and activation of retinoic acid-inducible gene I (RIG-I)-like signaling pathways [6]. Furthermore, the activation of the RIG-Ilike pathway increased the expression levels of DNA damage ( $\mathrm{Y}-\mathrm{H} 2 \mathrm{AX}, \mathrm{RAD} 51$, and PARP1) and apoptosis related proteins (BAX/BCL2 and Caspase-3) [6]. It is likely that ZEA and its metabolites increase ROS levels by interfering with mitochondrial oxidative phosphorylation [31, 32]. Interestingly, applying new single-cell RNA sequencing (scRNA-seq) technology, Tian et al. recently suggested that altered oxidative phosphorylation and DNA damage in oocytes can affect PF formation after ZEA exposure. In fact, they showed that maternal exposure to ZEA changed the developmental status of mouse oocytes and granulosa cells in offspring ovaries and altered the expression of genes associated with intercellular communication, oxidative phosphorylation, and DNA damage in oocytes [33]. Besides ROS generation, damage to mitochondrial activities can deregulate other relevant molecular pathways, including those involved in general cellular metabolism and gene expression [34,35]. Currently, the impact of mitochondrial dysfunction in epigenetics is emerging, but our understanding of this relationship and its effect on gene expression remains incomplete [36-38]. Interestingly, Zhang et al. showed that ZEA exposure of newborn mouse ovaries during in vitro culture caused a markedly higher level of DNA methylation at the $\mathrm{CpG}$ site of $\angle h x B$, a master gene for follicle formation, and accordingly decreased its expression [39].

In this context, the aim of this study was to further investigate the molecular mechanisms of the effect of ZEA on PF formation starting from scRNA-seq analyses of the cell populations of offspring mouse ovaries from mothers exposed to ZEA during the critical period of PF assembly. Focus was placed on the alteration of mitochondrial activities in oocytes leading to changes in metabolites associated with relevant epigenetic histone modifications and consequent gene expression.

\section{Materials And Methods}

\section{Animals and treatment}

C57/BL6 mouse breeding conditions and treatment methods in this experiment strictly complied with the Animal Care and Ethical Committee of Qingdao Agricultural University. The mice were bred in a house with a temperature of $23^{\circ} \mathrm{C}, 12 \mathrm{~h}$ light/dark cycles (lights off at 19:00 h), and adequate water and feed. Mating was arranged at about 17:00 $\mathrm{h}$, and the presence of a vaginal plug in females the next morning was viewed as a successful mating, which was considered as E0.5. 
In the present study, ZEA (Sigma, Z2125, MO, USA) was diluted to $40 \mathrm{mg} / \mathrm{ml}$ using DMSO, stored at -20 ${ }^{\circ} \mathrm{C}$, and diluted to $40 \mu \mathrm{g} / \mathrm{ml}$ with PBS before use. The dose of ZEA was chosen based on the study by Liu et al [30]. At E16.5, the mother mice were exposed to ZEA orally at a dose of $40 \mu \mathrm{g} / \mathrm{kg}$ body weight (BW) until PD0 or PD3 and were termed the ZEA group. Mother mice that were not treated were called the CTRL group. Subsequently, in order to conduct ZEA toxicity rescue, at E17.0, a part of ZEA group was orally taken CoQ10 (Sigma, C9538) at a dose of $20 \mathrm{mg} / \mathrm{kg} \mathrm{BW}$, which was dissolved in corn oil and called ZEA+ CoQ10 group. To eliminate the influence of the corn oil on the experimental results, one part of the control group was orally taken corn oil (the solvent of CoQ10) according to body weight and was designated as the corn oil (CO) group. At the same time, corn oil was also administered to one part of the ZEA group, which was designated as the ZEA+CO group. The dose of CoQ10 was chosen based on a previous study [40].

\section{Sample collection}

Sample preparation for sequencing: The method was the same as previously described [33]. Sample preparation for the experiment: At PD0 and PD3, ovaries were separated from mouse ovarian capsules in $0.9 \%$ normal saline. For the two ovaries from the same mouse, one was collected and cryopreserved at $-80{ }^{\circ} \mathrm{C}$, and the other one was fixed overnight at $4{ }^{\circ} \mathrm{C}$ in $4 \%$ paraformaldehyde (PFA, Solarbio, P1110, Beijing, China).

\section{scRNA-seq libraries and sequencing}

We used the single cell system of the $10 \times$ Genomics platform to obtain gel bead emulsions (GEMs). The 10x Genomics Chromium Single Cell 3' library and Gel Bead Kit v3 was applied for reverse transcription, barcode, and library establishment, according to the manufacturer's protocol. Paired-end sequencing was performed using an Illumina HiSeq X Ten instrument from Beijing Novogene Bioinformatics Co., Ltd., China. Raw data were then analyzed by CellRanger v.3.1.0 software, and the gene expression matrixes of four ovarian samples were obtained.

\section{Analysis of scRNA-seq data}

The "DoubleFinder" package was used to filter out abnormal cells (McGinnis et al. 2019), then "Seurat" was used for normalization, integration, and dimensionality reduction of the four sample sets. By using the "RunTSNE" function, the cells were divided into several clusters, and the cell types were determined according to the classic cell markers. The target cell type (oocyte) was extracted for further mining and analysis. The "FindMarkers" function was used to find the differential genes between the ZEA and CTRL groups. Further, metascape (https://metascape.org/gp/index.html) and GO packages were performed for enrichment analysis.

\section{Newborn ovary culture in vitro and RNA interference (RNAi)}

Ovaries isolated from PD0 mice were cultured for 3 days as previously described [9]. The isolated ovaries were cultured in 24-well culture dishes (NEST Biotechnology, Wuxi, China) in ovarian medium, which contained DMEM/F12 (Hyclone, SH30023.01B, Beijing, China), a-MEM (Hyclone, SH30265.01B) (1:1), 
$10 \%$ fetal bovine serum (FBS; Gibco, 10099-141, USA), $0.23 \mathrm{mmol} / \mathrm{L}$ sodium pyruvate (Hyclone, SH4000312), $100 \mathrm{IU} / \mathrm{ml}$ of penicillin $\mathrm{G}$, and $100 \mathrm{mg} / \mathrm{ml}$ of streptomycin sulfate (Hyclone, SV30010). Aliquots of 30 $\mu \mathrm{M}$ ZEA were introduced during in vitro culture to establish the ZEA treatment group, the dose of ZEA was based on the study by Zhang et al. [39]. Following the previous laboratory protocol, the siRNA of Hdac2 was transfected to silence gene expression [6,33]. Reagents used for transfection included:

Lipofectamine $^{\text {TM }} 3000$ Transfection Reagent (Lipo 3000, Thermo Fisher Scientific, L3000008, Germany) and $20 \mu \mathrm{mol} / \mathrm{L}$ si-Hdac2 (GenePharma, Shanghai, China; Table S1). The ovaries were cultured in a $37^{\circ} \mathrm{C}$, $5 \% \mathrm{CO}_{2}$ incubator for three days. The medium was changed for the first time $8 \mathrm{~h}$ after transfection, and again one day later.

\section{Immunostaining of ovarian sections}

The ovaries were fixed in 4\% PFA for $8 \mathrm{~h}$, washed with flowing water, dehydrated, and embedded in paraffin. Then sections were prepared with a thickness of $5 \mu \mathrm{m}$. Before staining, the histological sections underwent xylene dewaxing, gradient rehydration, and antigen retrieval. Subsequently, histological sections were used for immunofluorescence (IF) and immunohistochemistry (IHC). For IF, after antigen retrieval and natural cooling, the blocking buffer (containing 10\% goat serum diluted and 3\% BSA) was used to block the histological sections at room temperature for $45 \mathrm{~min}$; they were subsequently incubated overnight with the primary antibody (Table S2) at $4{ }^{\circ} \mathrm{C}$. Incubation with the secondary antibody (Table S2) took place at $37^{\circ} \mathrm{C}$ for $40 \mathrm{~min}$ in a dark room the next morning. Hoechst 33342 (Beyotime, C1022, Shanghai, China) or PI (Solarbio, P8080) was used for 3 min at $37^{\circ} \mathrm{C}$ to stain the nuclei. Finally, the slides were sealed with an anti-fluorescence attenuation quenching agent, before observation of sections under a fluorescence microscope (Olympus, BX51, Japan). For IHC, the same procedure took place as given above; however, before the blocking step, $3 \% \mathrm{H}_{2} \mathrm{O}_{2}$ was used to treat samples for $10 \mathrm{~min}$. After incubation with primary antibody, each slide was painted with horseradish peroxidase (HRP)-conjugated goat antirabbit lgG (Beyotime, A0258) for $45 \mathrm{~min}$ at $37^{\circ} \mathrm{C}$. Subsequently, DAB peroxidase substrate (ZSGB-BIO, ZLI-9017, Beijing, China) was used to create a chromogenic reaction, the cell nucleus was stained with hematoxylin, and finally after dehydration with alcohol and permeabilization with xylene, the histological sections were sealed with neutral resin and observed under a BX51 microscope.

\section{Western blotting}

The collected ovarian samples were taken from the $-80{ }^{\circ} \mathrm{C}$ refrigerator. The appropriate amount of RIPA (Beyotime, P0013C) was added to lyse ovarian tissues on ice for $20 \mathrm{~min}$; SDS-PAGE Sample Loading Buffer (5x, Beyotime, P0015L) was supplemented and the sample was boiled for $5 \mathrm{~min}$ to denature the protein. The $8-15 \%$ SDS-PAGE gel electrophoresis was applied according to the molecular weight of the target protein at $100 \mathrm{~V}$ constant voltage for 90-130 min. Then the target proteins were transferred onto a polyvinylidene fluoride membrane (PVDF; Millipore, ISEQ00010, USA) at a constant current of $300 \mathrm{~mA}$ for 120 min. Subsequently, the protein bands were blocked in TBST solution containing $5 \%$ BSA for $4 \mathrm{~h}$ on a low temperature shaker. Subsequently, the protein bands were incubated with the primary antibody (Table S2) overnight at $4^{\circ} \mathrm{C}$ and secondary antibody at room temperature for $75 \mathrm{~min}$. After rinsing in TBST, a 
BeyoECL Plus kit (Beyotime, P0018) was used for chemiluminescence. AlphaView SA software was used to perform the gray analysis of protein bands.

\section{Acetyl-CoA measurement}

A Mouse Acetyl Coenzyme A (ACA) ELISA Kit (JM-1689M2, Jingmei Biological, Jiangsu, China) was used to detect ACA levels. Accordingly, $25 \mathrm{mg}$ of fresh mouse ovaries were collected and lysed in PBS for 30 min. Following the standard requirements of the testing kit, the enzyme-labeled reagents were incubated at $37^{\circ} \mathrm{C}$ for $60 \mathrm{~min}$, and the treatment of color-developing solution was performed for 15 min under dark conditions. Finally, the stop solution was added to terminate the reaction, and the absorbance value at a wavelength of $450 \mathrm{~nm}$ was obtained with a microplate reader within $15 \mathrm{~min}$, which was used for the calculation of ACA content.

\section{Citrate measurement}

A Citric acid (CA) content detection kit (Solarbio, BC2150) was used to detect the level of CA. For each test, $25 \mathrm{mg}$ of ovarian tissue was prepared, and the reagents were used in strict accordance with the kit instructions. After standing at room temperature for $30 \mathrm{~min}$, a microplate reader was used to detect absorbance at the wavelength of $545 \mathrm{~nm}$.

\section{Transmission Electron Microscopy (TEM)}

Fresh ovaries were fixed overnight with $2.5 \%$ glutaraldehyde at $4{ }^{\circ} \mathrm{C}$. According to standard TEM procedures, the samples were embedded in resin [41]. Serial sectioning with an EM UC7 ultramicrotome $(50 \mathrm{~nm})$ was conducted, and the ultrathin sections were stained with lead citrate and uranyl acetate for follow-up observation with HT7700 TEM (Hitachi, Tokyo, Japan).

\section{Detection of mitochondrial membrane potential}

Mitochondrial membrane potential of the fresh ovaries was detected using a JC-1 detection kit (Beyotime, C2006). Using trypsin (Sangon, A003702, Shanghai, China) and collagenase (Sigma, C5138, 9:1), the ovaries were digested into single cells at $37^{\circ} \mathrm{C}(30 \mathrm{~min})$, and the samples were incubated with a JC-1 staining working solution at $37^{\circ} \mathrm{C}(20 \mathrm{~min})$. After washing with a JC-1 staining buffer, the samples were diluted and tested for flow cytometry (BD Calibur, CA, USA). Finally, results were analyzed with FlowJo_v.10 software.

\section{Real-Time quantitative PCR (RT-qPCR)}

Total RNA was obtained from mouse ovaries with a SPARKeasy Improved Tissue/cell RNA kit (Sparkjade, AC0202, Shandong, China). The extracted RNA was subjected to reverse transcription to synthesize cDNA with a SPARKscript II RT plus kit (With gDNA Eraser) (Sparkjade, AG0304). RT-qPCR was performed using SYBR Premix Ex Taq ${ }^{\text {TM }}$ II (Vazyme, Q711-02, Nanjing, China) with a Roche 480 (Roche, Germany) Light Cycler RT-qPCR instrument. All primers are listed in Table S1.

\section{Statistical analysis}


All experiments had at least three repetitions between groups. The data were presented as mean \pm SD, and the significance of differences between groups was analyzed using the t-test or ANOVA and Graph Pad Prism 8.0 software. $P<0.05\left(^{*}\right)$ represents a significant difference, and $P<0.01\left({ }^{\star *}\right)$ indicates a remarkably significant difference.

\section{Results}

\section{scRNA-seq identified six cell types in the ovaries of newborn pups from either CTRL or ZEA exposed mothers}

In order to explore the effect of the exposure of pregnant mothers to ZEA on the transcriptome of newborn mouse ovaries, the compound was orally administered $(40 \mu \mathrm{g} / \mathrm{kg})$ from E16.5 until PD0 or PD3. Offspring ovaries were collected at PD0 or PD3 for scRNA-seq analyses (Fig. 1A). After data preprocessing and quality control, a total of 16468 ovarian cells were analyzed (PDO: CTRL, 4004; ZEA, 4436. PD3: CTRL, 3659; ZEA, 4369; Table S3). The four samples were integrated, and according to tdistributed stochastic neighbor embedding (tSNE), 18 cell clusters and six distinct cell types were identified both in CTRL and ZEA samples (Figs. 1B and 1D, S1A and S1B, Table S4). These included five oocyte clusters (Dazl and Dppa3), six pregranulosa cell clusters (Fst and Amhr2), four interstitial cell clusters (Mfap4 and Col1a1), one erythrocyte cluster (Alas2 and Rhd), one immune cell cluster (Tyrobp and Cd52), and one endothelial cell cluster (Egfl7 and Ap/nr) (Figs. 1C, S1C) [5]. Likewise, when uniform manifold approximation and projection (UMAP) technology was used, 18 cell clusters and six cell types were also identified (Fig. S1D and S1E).

\section{Maternal exposure to ZEA impaired PF formation in the newborn ovaries}

In order to verify the effect of maternal ZEA exposure more accurately on offspring PF formation, we focused on the transcription dynamic of oocytes. First, by extracting oocytes from four sample sets, tSNE projection subdivided the oocytes into 10 subclusters (Figs. 2A, S2A upper). On this basis, we classified oocytes as pre- (Meioc and Malat1), early- (Figla and Lhx8), and late- (Padi6 and Zp3) follicular stages (Fig. S2A below and S2B) [23]. The violin plot results showed that in oocytes the transcripts of key genes crucial for PF formation such as Syce3, Nobox, Lhx8, and Padi6 significantly decreased in PD0 and PD3 ovaries after ZEA exposure (Fig. 2B). In addition, the staining of ovary sections for the germ cell specific $\mathrm{MVH}$ protein showed that ZEA exposure caused a significant reduction of oocyte number at PD3 (CTRL = $272.42 \pm 16.15, \mathrm{ZEA}=186.21 \pm 7.31 ; P<0.01)$, but the oocyte number at PD0 has no significant difference, and that the percentage of oocytes within follicles was also decreased compared with those in germ cell cysts both at PD0 and PD3 (PD0: CTRL $=29.92 \pm 0.94 \%, Z E A=20.62 \pm 1.29 \% ; P<0.05$. PD3: $C T R L=48.73 \pm 1.17 \%, Z E A=38.49 \pm 1.61 \% ; P<0.01$. Fig. $2 C$ and $2 D)$. WB analyses also confirmed downregulation of MVH and LHX8 in the PD3 ovaries of offspring from ZEA exposed mothers; moreover, the LHX8 was also reduced in PD0 after ZEA exposed mothers (Fig. 2E and 2F). 
Altogether, these results confirmed the notion that maternal exposure to ZEA during the period of folliculogenesis in the fetal ovaries had negative impacts on PF formation, including deregulating the expression of key genes involved in this process and increasing the rate of oocyte degeneration $[6,30]$.

\section{ZEA exposure dysregulated the expression of genes encoding proteins involved in mitochondrial processes in oocytes}

To dissect the underlying molecular mechanism behind ZEA exposure impairing PF formation, we performed differential expression gene (DEG) analysis on oocytes in the investigated periods. The results showed that at PD0, maternal ZEA exposure resulted in down-regulation of 134 and up-regulation of 194 genes in the oocytes of offspring ovaries; at PD3, the number of down-regulated genes was 210 and that of up-regulated increased to 196 (Fig. S3A and S3B, Table S5). Metascape enrichment analysis of the $D E G s$ revealed that mitochondrial electron transport was one of the processes that was greatly affected by ZEA exposure (Fig. S3A and S3B).

When oocytes were subdivided into pre-, early-, and late-follicular stages (Fig. S2A below and S2B), ZEA treatment resulted in 329 DEGs (152 down- and 177 up-regulated) at PD0 and 22 DEGs (13 down- and 9 up-regulated) at PD3 in the pre-PF stage (Table S6; Fig. 3A left). For the early-PF stage (Table S6), there were 346 DEGs (212 down- and 134 up-regulated) at PD0, and 433 DEGs (217 down- and 216 upregulated) at PD3 (Fig. 3A middle); and for the late-PF stage (Table S6), there were 434 DEGs (275 downand 159 up-regulated) at PD0 and 402 DEGs (202 down- and 200 up-regulated) at PD3 (Fig. 3A right). At each stage, there were 20,219, and 247 DEGs between PD0 and PD3, respectively (Fig. 3B). According to the enrichment analysis, most of the affected biological processes were again associated with mitochondrial activities (Fig. 3C).

\section{ZEA exposure affected the structure and molecular composition of oocyte mitochondria and mitochondrial activities in ovaries}

To confirm the results of the scRNA-seq analyses concerning the effects of ZEA on mitochondrial processes in oocytes, we next performed morphological, molecular, and biochemical evaluations of the mitochondria in such cells. TEM observations showed frequent morphological alterations such as fragmentation and vacuolization of mitochondria in oocytes after ZEA exposure (Fig. 4A). Data from scRNA-seq revealed that the transcripts of many proteins of the electron transport chain (ETC) complexes such NDUFB2, SDHB, COX6A1, and ATP5L, were down-regulated in oocytes following ZEA treatment (Fig. 4B); Western blot analyses on the whole ovaries confirmed these data at a protein level (Fig. 4C). The mitochondrial membrane potential (MMP), however, did not appear to be significantly different between CTRL and ZEA groups of ovaries (Fig. S2C), implying a substantial integrity of mitochondria. 
Finally, we used targeted mitochondrial metabolite analyses to monitor changes of mitochondrial activities. Since the level of ACA and CA can reflect tricarboxylic acid cycle (TCA cycle) flux and ETC activities [36], we measured the levels of these compounds in offspring ovaries. The levels of both ACA and CA were significantly lower in the ovaries of offspring from ZEA exposed mice (Fig. 4D and 4E).

\section{ZEA induced oocyte autophagy in PD3 ovaries}

Much evidence indicates that ACA levels are important regulators of autophagy [42-45]. Thus, we next

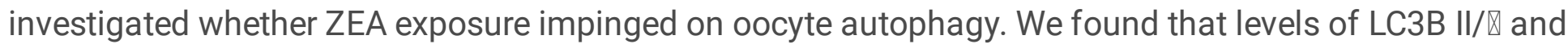
p62, which are often used as biomarkers of ongoing autophagy, increased in ovaries of PD3 offspring from ZEA exposed mothers, whereas those of $\mathrm{p}-\mathrm{mTOR}$, a negative regulator of autophagy, were decreased in comparison to CTRL (Fig. 5A and 5B). IF staining showed more LC3B dots (an indication of autophagy degree) in the cytoplasm of oocytes within ovaries of PD3 offspring from ZEA exposed mothers (Fig. 5C and 5D). Accordingly, TEM observations revealed autophagosomes in oocytes of these ovaries (Fig. 5E).

\section{ZEA altered the expression of KAT2A, HDAC2, and SIRT1 in oocytes, and histone acetylation in offspring ovaries}

Since the activity of histone acetyl-transferases (HATs), histone deacetylases (HDACs), and consequently the level of histone acetylation depend on ACA and CA availability [37, 46-48], the levels of transcripts for the KAT2A lysine histone acetylase and the HDAC2 and SIRT1 histone deacetylases in offspring ovaries were investigated. The results showed that the RNA levels of Kat2a were down-regulated whereas those of Hdac2 and Sirt1 were up-regulated in PD3 offspring ovaries from ZEA exposed mothers in comparison to CTRL (Fig. S4A). In line with these observations, western blot showed an up-regulation of both HDAC2 and SIRT1 and a down-regulation of KAT2A proteins in offspring ovaries of ZEA exposed mothers (Figs. $6 \mathrm{~A}$, Fig. S4B). Moreover, HDAC2 was immunolocalized mainly in the nuclei of both oocytes and pregranulosa cells in either condition (Fig. 6B). To determine which histones were affected by ZEA exposure, the expression levels of H3K9ac, H3K23ac, H4K12ac, and H4K16ac were tested. Western blot showed that the levels of H3K9ac and H4K12ac were decreased while those of H3K23ac and H4K16ac remained unaffected in the offspring ovaries of ZEA exposed mothers (Figs. 6C, S4E).

\section{Inhibition of Hdac2 expression alleviated the deleterious effect of ZEA on the cultured ovaries}

HDAC2 has been reported to play a crucial role in early folliculogenesis and oocyte development [7, 4954]. Furthermore, based on the results reported above, we speculated that upregulated HDAC2 and the subsequent deregulation of the acetylation of some histones such as H3K9ac and H4K12ac might be a critical consequence of the impairment of mitochondrial activities in oocytes caused by ZEA, and that such conditions increased autophagia in oocytes and decreased their capacity to form PFs. To verify such possibilities, RNAi was then used to down-regulate the expression of Hdac2 in cultured newborn 
ovaries. For this, PD0 ovaries were cultured for three days in the presence or absence of $30 \mu \mathrm{M} Z \mathrm{ZA}$ and with or without si-Hdac2, and levels of the oocyte LHX8 and MVH proteins, H3K9ac and H4K12ac, and the autophagy marker LC3B were determined by WB; at the same time, the percentage of oocytes within PFs in tissue sections was evaluated. The results showed that the amount of HDAC2 was significantly reduced in the presence of si-Hdac2 alone compared to CTRL, as expected (Fig. 6D). Moreover, and most importantly, the expression levels of LHX8 and MVH (Fig. 6E and 6F), of H3K9ac and H4K12ac (Fig. 6G and $6 \mathrm{H}$ ), the numbers of oocytes and the percentage of oocytes in PFs (Fig. S4C and S4D), nearly reverted to those of CTRL. Moreover, ovaries cultured in ZEA+si-Hdac2 had significantly lower LC3B II/ $\mathbb{Z}$ levels than ovaries cultured in the presence of $30 \mu \mathrm{M}$ ZEA (Fig. $6 \mathrm{G}$ and $6 \mathrm{I}$ ).

\section{Oral administration of CoQ10 alleviated most of the effects of ZEA on offspring ovaries}

CoQ10 is an important electron donor that can transport electrons from complexes I and II (received from the TCA cycle) to complex III in the mitochondrial ETC [40]. CoQ10 is essential for mitochondrial activities as point mutations in enzymes responsible for CoQ10 synthesis can alter phenotypes in the high energyconsuming tissues that can be partially alleviated by oral administration of CoQ10 [55-57].

To verify whether ZEA-induced oocyte mitochondrial dysfunction is responsible for the effects of the drug on the offspring ovaries described here, we tested whether CoQ10 counteracted such effects. For this, 20 $\mathrm{mg} / \mathrm{kg}$ CoQ10 was orally administrated to pregnant mothers at $12 \mathrm{~h}$ after each ZEA dosing and PD3 offspring ovaries were analyzed for various parameters (Fig. 7A). Western blot analyses showed that in the offspring ovaries from ZEA+CoQ10 mothers, the levels of MVH, LHX8, and the ETC proteins COX6A1, HDAC2, and LC3B were similar to those of controls (Fig. 7B and 7C). Moreover, while the numbers of oocytes were similar in all experimental groups, CoQ10 administration restored the percentage of oocytes enclosed within follicles, decreased by ZEA, to the control values (CO $=50.26 \pm 1.13 \%$, ZEA+CO $=60.33 \pm$ $1.02 \%$, ZEA+CoQ10 $=49.71 \pm 3.16 \%$, CoQ10 $=52.09 \pm 4.04 \% ; P<0.01$ (Fig. 7D and 7E)

\section{Discussion}

scRNA-seq technology is capable of identifying cell heterogeneity in tissue and organs, which allows many novel insights in our understanding of their biology $[58,59]$. This technology has recently been applied to describe the transcriptome landscape of gametogenic processes such as PF formation $[2,5$, 23]. In a previous study, using scRNA-seq, we were able to detect six cell types in developing mouse ovaries including oocytes, pregranulosa cells, interstitial cells, erythrocytes, immune cells, and endothelial cells and to gain new information about the mechanisms guiding early folliculogenesis [23]. In the present paper, we used this technology to identify processes in oocytes affected by ZEA exposure that negatively affected their capability to orchestrate PF formation. Significantly, our data showed that exposure of pregnant mice to ZEA reduced the expression of transcripts of key genes known to be crucial for PF formation such as Syce3, Nobox, Lhx8, and Padi6 in the oocytes of offspring ovaries. Furthermore, in such ovaries, the number and proportion of oocytes enclosed in follicles were also significantly 
decreased. Thus, our research confirmed previously reported results that ZEA exposure has a negative impact on PF formation in fetal ovaries during the early period of folliculogenesis $[6,30]$.

For the processes affected by ZEA in oocytes, functional enrichment of DEGs pointed to the reduced expression of proteins with a role in the mitochondrial ETC. Considering the low expression of phosphofructokinase, oocytes mainly depend on the mitochondrial ETC and TCA to produce ATP; therefore, these processes are crucial for oogenesis [60]. Mitochondrial alterations in oocytes exposed to ZEA were confirmed by morphological and further scRNA-seq bioinformatics analyses. These results together with the findings that the levels of metabolites such as ACA and CA were significantly reduced while autophagic markers were increased in the ovaries of offspring from ZEA exposed mothers, supported the notion of mitochondrial dysfunction in the oocytes of these ovaries as the main cause of PF assembly impairment. Several previous studies report that the level of ACA reflects the general energetic state of cells and is inversely proportional to autophagy [43]. In line with these, here we found that autophagy was increased in the ovaries from PD3 offspring. Epigenetic changes in the genome are emerging as relevant consequences of mitochondrial dysfunction, and ACA and CA availability are crucial for the activity of the major regulators of histone acetylation such as HATs and HDACs [37, 61, 62]; therefore, we next decided to investigate the levels of transcripts for the KAT2A lysine histone acetylase and the HDAC2 and SIRT1 histone deacetylases in offspring ovaries. Relevant in this regard, HDACs are reported to play a crucial role in early folliculogenesis and oocyte development $[49-52,63]$. The results showed that the mRNA and protein levels of KAT2A were downregulated whereas those of HDAC2 and SIRT1 were upregulated in PD3 offspring ovaries from ZEA exposed mothers in comparison to control groups. Moreover, we identified H3K9ac and H4K12ac as histones whose ovarian levels were negatively affected by ZEA. The fact that neutralizing Hdac2 mRNA restored, nearly to control levels, the expression of LHX8 and MVH, LC3B, H3K9ac, and H4K12ac, as well as PF number in newborn ovaries cultured in the presence of ZEA, strongly implied that misregulation of the activity of this deacetylase was mostly responsible for the deleterious effects of ZEA on PF assembly. In line with these findings, Li et al. have reported that the administration of trichostatin A, an inhibitor of HDAC, to female mice alleviates ovarian structural damage and decreases autophagy caused by cigarette smoking [7].

Mitochondrial function is necessary for oogenesis, fertilization, and embryonic development [64-66]. Moreover, mitochondrial dysfunction is accompanied by a decrease in ATP and CA levels, which are closely related to oocyte insufficiency and decreased fertilization rate $[65,67,68]$. Consistent with this, it has also been found in human clinical studies that mitochondrial function is closely related to reproductive ability $[69,70]$. Interestingly, a recent study showed that gut microbiota improved fertility in Drosophila melanogaster by supplementing riboflavin (a precursor of FAD and FMN, two essential coenzymes in mitochondria). Relevant to the present paper, this also restored ovarian reserve [34]. In addition, in vivo supplementation of nicotinamide mononucleotide (NMN, an essential co-enzyme in mitochondria) or CoQ10, an important hydrogen transporter in the ETC, increased ovulatory potential, and oocyte quantity and quality in aged mice $[40,64,71]$. Moreover, CoQ10 supplementation is beneficial for polycystic ovarian syndrome (PCOS) patients to improve their mature oocyte count [72]. On these bases, we here attempted to attenuate the effects of ZEA by improving mitochondrial function using CoQ10. The 
oral administration of CoQ10 to pregnant mice prevented most of the deleterious effects of ZEA on offspring ovaries including reduced expression of MVH and the ETC COX6A1, up-regulation of HDAC2, and LC3B, and impairment of PF assembly.

In conclusion, our data support the notion that the impairment of PF assembly caused by ZEA in postnatal mouse ovaries is mainly a consequence of mitochondrial dysfunction, which leads to increased autophagy and epigenetic changes primarily in oocytes (Fig. 8). Moreover, the effectiveness of CoQ10 in mitigating the effects of ZEA suggest a means of counteracting the deleterious effects of this compound on early folliculogenesis in mammals.

\section{Abbreviations}

ACA: acetyl coenzyme A

BW: body weight

CA: citric acid

CoQ10: coenzyme Q10

DEG: differential expression gene

E17.5: embryonic 17.5 day

E2: $17 \beta$-estradiol

ETC: electron transport chain

GEMs: gel bead emulsions

HAT: histone acetyltransferase

HDAC2: histone deacetylase 2

HRP: horseradish peroxidase

IF: immunofluorescence

IHC: immunohistochemistry

KAT2A: lysine acetyltransferase $2 \mathrm{~A}$

MMP: mitochondrial membrane potential

OR: ovarian reserve 
PCOS: polycystic ovarian syndrome

PD0: postnatal 0 day

PF: primordial follicle

PFA: paraformaldehyde

PVDF: polyvinylidene fluoride membrane

RT-qPCR: Real-Time quantitative PCR

RNAi: RNA interference

ROS: reactive oxygen species

scRNA-seq: single-cell RNA sequencing

TCA: tricarboxylic acid

tSNE: t-distributed stochastic neighbor embedding

TEM: transmission electron microscopy

UMAP: uniform manifold approximation and projection

ZEA: zearalenone

\section{Declarations}

CRediT authorship contribution statement Wei Shen, Lan Li and Jun-Jie Wang designed the study. YanQin Feng, Ming-Hao Li performed the experiments for this work. Yu Tian analyzed the single-cell RNA sequence data in this study. Yan-Qin Feng, Jun-Jie Wang and Massimo De Felici wrote the manuscript. Lan Li and Ai-Hong Zhao provided funding. All authors contributed to discussion of the study and revision of this manuscript.

Acknowledgements This work was supported by Science \& Technology Fund Planning Projects of Qingdao City (21-1-4-ny-7-nsh), Natural Science Foundation of Shandong Province (ZR202103020217) and Taishan Scholar Foundation of Shandong Province (ts20190946).

Conflict of interest The authors declare that they have no conflict of interest.

Ethics approval C57/BL6 mouse breeding conditions and treatment methods in this experiment strictly complied with the Animal Care and Ethical Committee of Qingdao Agricultural University. 


\section{References}

1. Wang S, Zheng Y, Li J, Yu Y, Zhang W, Song M et al (2020) Single-Cell Transcriptomic Atlas of Primate Ovarian Aging. Cell. ;180(3):585-600 e19. doi: 10.1016/j.cell.2020.01.009. PubMed PMID: 32004457

2. He Y, Chen Q, Dai J, Cui Y, Zhang C, Wen X et al (2021) Single-cell RNA-Seq reveals a highly coordinated transcriptional program in mouse germ cells during primordial follicle formation. Aging Cell 20(7):e13424. doi: 10.1111/acel.13424. PubMed PMID: 34174788; PubMed Central PMCID: PMCPMC8282241

3. Wang C, Zhou B, Xia G (2017) Mechanisms controlling germline cyst breakdown and primordial follicle formation. Cell Mol Life Sci 74(14):2547-2566. doi: 10.1007/s00018-017-2480-6. PubMed PMID: 28197668

4. Niu W, Spradling AC (2020) Two distinct pathways of pregranulosa cell differentiation support follicle formation in the mouse ovary. Proceedings of the National Academy of Sciences. ;117(33):20015-26. doi: 10.1073/pnas.2005570117

5. Wang JJ, Ge W, Zhai QY, Liu JC, Sun XW, Liu WX et al (2020) Single-cell transcriptome landscape of ovarian cells during primordial follicle assembly in mice. PLoS Biol 18(12):e3001025. doi: 10.1371/journal.pbio.3001025. PubMed PMID: 33351795; PubMed Central PMCID: PMCPMC7787681

6. Kong L, Zhao AH, Wang QW, Feng YQ, Yan ZH, Li MH et al (2021) Maternal Zearalenone exposure impacted ovarian follicle formation and development of suckled offspring. Sci Total Environ 788:147792. doi: 10.1016/j.scitotenv.2021.147792. PubMed PMID: 34134368

7. Li F, Ding J, Cong Y, Liu B, Miao J, Wu D et al (2020) Trichostatin A alleviated ovarian tissue damage caused by cigarette smoke exposure. Reprod Toxicol 93:89-98. doi: 10.1016/j.reprotox.2020.01.006. PubMed PMID: 31987896

8. Zhang MY, Tian Y, Yan ZH, Li WD, Zang CJ, Li L et al (2020) Maternal Bisphenol S exposure affects the reproductive capacity of F1 and F2 offspring in mice. Environ Pollut 267:115382. doi: 10.1016/j.envpol.2020.115382. PubMed PMID: 32866863

9. Liu JC, Li L, Yan HC, Zhang T, Zhang P, Sun ZY et al (2019) Identification of oxidative stress-related Xdh gene as a di(2-ethylhexyl)phthalate (DEHP) target and the use of melatonin to alleviate the DEHP-induced impairments in newborn mouse ovaries. J Pineal Res 67(1):e12577. doi: 10.1111/jpi.12577. PubMed PMID: 30938853

10. da Costa CS, Oliveira TF, Freitas-Lima LC, Padilha AS, Krause M, Carneiro M et al (2021) Subacute cadmium exposure disrupts the hypothalamic-pituitary-gonadal axis, leading to polycystic ovarian syndrome and premature ovarian failure features in female rats. Environ Pollut 269:116154. doi: 10.1016/j.envpol.2020.116154. PubMed PMID: 33280922

11. Gruber-Dorninger C, Jenkins T, Schatzmayr G (2019) Global Mycotoxin Occurrence in Feed: A TenYear Survey. Toxins (Basel) 11(7):375 : 10.3390/toxins11070375. PubMed PMID: 31252650; 
PubMed Central PMCID: PMCPMC6669473

12. Rai A, Das M, Tripathi A (2019) Occurrence and toxicity of a fusarium mycotoxin, zearalenone. Crit Rev Food Sci Nutr 60(16):2710-2729. doi: 10.1080/10408398.2019.1655388

13. Ropejko K, Twaruzek M (2021) Zearalenone and Its Metabolites-General Overview, Occurrence, and Toxicity. Toxins (Basel) 13(1):35 : 10.3390/toxins13010035. PubMed PMID: 33418872; PubMed Central PMCID: PMCPMC7825134

14. Zhu L, Yuhan J, Huang K, He X, Liang Z, Xu W (2021) Multidimensional analysis of the epigenetic alterations in toxicities induced by mycotoxins. Food Chem Toxicol 153:112251. doi: 10.1016/j.fct.2021.112251. PubMed PMID: 33961929

15. Rajendran P, Ammar RB, Al-Saeedi FJ, Mohamed ME, ElNaggar MA, Al-Ramadan SY et al (2020) Kaempferol Inhibits Zearalenone-Induced Oxidative Stress and Apoptosis via the PI3K/Akt-Mediated Nrf2 Signaling Pathway: In Vitro and In Vivo Studies. Int J Mol Sci 22(1):217. doi: 10.3390/ijms22010217. PubMed PMID: 33379332; PubMed Central PMCID: PMCPMC7794799

16. Yang D, Jiang X, Sun J, Li X, Li X, Jiao R et al (2018) Toxic effects of zearalenone on gametogenesis and embryonic development: A molecular point of review. Food Chem Toxicol 119:24-30. doi: 10.1016/j.fct.2018.06.003. PubMed PMID: 29864477

17. Liu J, Applegate T (2020) Zearalenone (ZEN) in Livestock and Poultry: Dose, Toxicokinetics, Toxicity and Estrogenicity. Toxins 12(6):377. doi: 10.3390/toxins12060377

18. Zhao F, Li R, Xiao S, Diao H, El Zowalaty AE, Ye X (2014) Multigenerational exposure to dietary zearalenone (ZEA), an estrogenic mycotoxin, affects puberty and reproduction in female mice. Reprod Toxicol 47:81-88. doi: 10.1016/j.reprotox.2014.06.005. PubMed PMID: 24972337; PubMed Central PMCID: PMCPMC4137769

19. Gajecka M, Zielonka L, Gajecki M (2015) The Effect of Low Monotonic Doses of Zearalenone on Selected Reproductive Tissues in Pre-Pubertal Female Dogs--A Review. Molecules 20(11):2066920687. doi: 10.3390/molecules201119726. PubMed PMID: 26610443; PubMed Central PMCID: PMCPMC6331970

20. Tan S, Ge W, Wang J, Liu W, Zhao Y, Shen W et al (2020) Zearalenone-induced aberration in the composition of the gut microbiome and function impacts the ovary reserve. Chemosphere 244:125493. doi: 10.1016/j.chemosphere.2019.125493. PubMed PMID: 32050327

21. Liu JC, Yan ZH, Li B, Yan HC, De Felici M, Shen W (2021) Di (2-ethylhexyl) phthalate impairs primordial follicle assembly by increasing PDE3A expression in oocytes. Environ Pollut 270:116088. doi: 10.1016/j.envpol.2020.116088. PubMed PMID: 33234378

22. Liu WX, Donatella F, Tan SJ, Ge W, Wang JJ, Sun XF et al (2021) Detrimental effect of Bisphenol S in mouse germ cell cyst breakdown and primordial follicle assembly. Chemosphere 264(Pt 1):128445. doi: 10.1016/j.chemosphere.2020.128445. PubMed PMID: 33017704

23. Wang JJ, Tian Y, Li MH, Feng YQ, Kong L, Zhang FL et al (2021) Single-cell transcriptome dissection of the toxic impact of $\mathrm{Di}$ (2-ethylhexyl) phthalate on primordial follicle assembly. Theranostics 
11(10):4992-5009. doi: 10.7150/thno.55006. PubMed PMID: 33754040; PubMed Central PMCID: PMCPMC7978297

24. Chen Y, Breen K, Pepling ME (2009) Estrogen can signal through multiple pathways to regulate oocyte cyst breakdown and primordial follicle assembly in the neonatal mouse ovary. J Endocrinol 202(3):407-417. doi: 10.1677/JOE-09-0109. PubMed PMID: 19505948

25. Johansson HKL, Svingen T, Fowler PA, Vinggaard AM, Boberg J (2017) Environmental influences on ovarian dysgenesis - developmental windows sensitive to chemical exposures. Nat Rev Endocrinol 13(7):400-414. doi: 10.1038/nrendo.2017.36. PubMed PMID: 28450750

26. Huang D, Cui L, Sajid A, Zainab F, Wu Q, Wang X et al (2019) The epigenetic mechanisms in Fusarium mycotoxins induced toxicities. Food Chem Toxicol 123:595-601. doi:

10.1016/j.fct.2018.10.059

27. Zheng W, Wang B, Si M, Zou H, Song R, Gu J et al (2018) Zearalenone altered the cytoskeletal structure via ER stress- autophagy- oxidative stress pathway in mouse TM4 Sertoli cells. Sci Rep 8(1):3320. doi: 10.1038/s41598-018-21567-8. PubMed PMID: 29463855; PubMed Central PMCID: PMCPMC5820275

28. So MY, Tian Z, Phoon YS, Sha S, Antoniou MN, Zhang J et al (2014) Gene expression profile and toxic effects in human bronchial epithelial cells exposed to zearalenone. PLoS ONE 9(5):e96404. doi: 10.1371/journal.pone.0096404. PubMed PMID: 24788721; PubMed Central PMCID:

PMCPMC4008614

29. Tatay E, Espin S, Garcia-Fernandez AJ, Ruiz MJ (2017) Oxidative damage and disturbance of antioxidant capacity by zearalenone and its metabolites in human cells. Toxicol In Vitro 45( $\mathrm{Pt}$ 3):334-339. doi: 10.1016/j.tiv.2017.04.026. PubMed PMID: 28477956

30. Liu KH, Sun XF, Feng YZ, Cheng SF, Li B, Li YP et al (2017) The impact of Zearalenone on the meiotic progression and primordial follicle assembly during early oogenesis. Toxicol Appl Pharmacol 329:917. doi: 10.1016/j.taap.2017.05.024. PubMed PMID: 28552778

31. Ben Salem I, Boussabbeh M, Prola A, Guilbert A, Bacha H, Lemaire C et al (2016) Crocin protects human embryonic kidney cells (HEK293) from alpha- and beta-Zearalenol-induced ER stress and apoptosis. Environ Sci Pollut Res Int. ;23(15):15504-14. doi: 10.1007/s11356-016-6741-y. PubMed PMID: 27121014

32. Fan W, Shen T, Ding Q, Lv Y, Li L, Huang K et al (2017) Zearalenone induces ROS-mediated mitochondrial damage in porcine IPEC-J2 cells. J Biochem Mol Toxicol 31(10). doi:

10.1002/jbt.21944. PubMed PMID: 28608607

33. Tian Y, Zhang MY, Zhao AH, Kong L, Wang JJ, Shen W et al (2021) Single-cell transcriptomic profiling provides insights into the toxic effects of Zearalenone exposure on primordial follicle assembly. Theranostics 11(11):5197-5213. doi: 10.7150/thno.58433. PubMed PMID: 33859742; PubMed Central PMCID: PMCPMC8039963

34. Gnainsky Y, Zfanya N, Elgart M, Omri E, Brandis A, Mehlman T et al (2021) Systemic Regulation of Host Energy and Oogenesis by Microbiome-Derived Mitochondrial Coenzymes. Cell Reports 
34(1):108583. doi: 10.1016/j.celrep.2020.108583

35. Wang ZH, Liu Y, Chaitankar V, Pirooznia M, Xu H (2019) Electron transport chain biogenesis activated by a JNK-insulin-Myc relay primes mitochondrial inheritance in Drosophila. Elife 15(8):e49309 : 10.7554/eLife.49309. PubMed PMID: 31612862; PubMed Central PMCID: PMCPMC6809605

36. Zhu D, Wu X, Zhou J, Li X, Huang X, Li J et al (2020) NuRD mediates mitochondrial stress-induced longevity via chromatin remodeling in response to acetyl-CoA level. Sci Adv 6(31):eabb2529. doi: 10.1126/sciadv.abb2529. PubMed PMID: 32789178; PubMed Central PMCID: PMCPMC7400466

37. Sivanand S, Viney I, Wellen KE (2018) Spatiotemporal Control of Acetyl-CoA Metabolism in Chromatin Regulation. Trends Biochem Sci 43(1):61-74. doi: 10.1016/j.tibs.2017.11.004. PubMed PMID: 29174173; PubMed Central PMCID: PMCPMC5741483

38. Lozoya OA, Wang T, Grenet D, Wolfgang TC, Sobhany M, Ganini da Silva D et al (2019) Mitochondrial acetyl-CoA reversibly regulates locus-specific histone acetylation and gene expression. Life Sci Alliance 2(1):e201800228. doi: 10.26508/Isa.201800228. PubMed PMID: 30737248; PubMed Central PMCID: PMCPMC6369536

39. Zhang GL, Sun XF, Feng YZ, Li B, Li YP, Yang F et al (2017) Zearalenone exposure impairs ovarian primordial follicle formation via down-regulation of Lhx8 expression in vitro. Toxicol Appl Pharmacol 317:33-40. doi: 10.1016/j.taap.2017.01.004. PubMed PMID: 28089945

40. Ben-Meir A, Burstein E, Borrego-Alvarez A, Chong J, Wong E, Yavorska T et al (2015) Coenzyme Q10 restores oocyte mitochondrial function and fertility during reproductive aging. Aging Cell 14(5):887895. doi: 10.1111/acel.12368. PubMed PMID: 26111777; PubMed Central PMCID: PMCPMC4568976

41. Wang Y-Y, Sun Y-C, Sun X-F, Cheng S-F, Li B, Zhang X-F et al (2017) Starvation at birth impairs germ cell cyst breakdown and increases autophagy and apoptosis in mouse oocytes. Cell Death Dis 8(2):e2613-e. doi: 10.1038/cddis.2017.3

42. Pietrocola F, Galluzzi L, Bravo-San Pedro JM, Madeo F, Kroemer G (2015) Acetyl coenzyme A: a central metabolite and second messenger. Cell Metab 21(6):805-821. doi:

10.1016/j.cmet.2015.05.014. PubMed PMID: 26039447

43. Schroeder S, Pendl T, Zimmermann A, Eisenberg T, Carmona-Gutierrez D, Ruckenstuhl C et al (2014) Acetyl-coenzyme A: a metabolic master regulator of autophagy and longevity. Autophagy 10(7):1335-1337. doi: 10.4161/auto.28919. PubMed PMID: 24904996; PubMed Central PMCID: PMCPMC4203557

44. Eisenberg T, Schroeder S, Andryushkova A, Pendl T, Kuttner V, Bhukel A et al (2014) Nucleocytosolic depletion of the energy metabolite acetyl-coenzyme a stimulates autophagy and prolongs lifespan. Cell Metab 19(3):431-444. doi: 10.1016/j.cmet.2014.02.010. PubMed PMID: 24606900; PubMed Central PMCID: PMCPMC3988959

45. Marino G, Pietrocola F, Eisenberg T, Kong Y, Malik SA, Andryushkova A et al (2014) Regulation of autophagy by cytosolic acetyl-coenzyme A. Mol Cell 53(5):710-725. doi: 10.1016/j.molcel.2014.01.016. PubMed PMID: 24560926 
46. Moussaieff A, Rouleau M, Kitsberg D, Cohen M, Levy G, Barasch D et al (2015) Glycolysis-mediated changes in acetyl-CoA and histone acetylation control the early differentiation of embryonic stem cells. Cell Metab 21(3):392-402 PubMed PMID: 25738455

47. Reid MA, Dai Z, Locasale JW (2017) The impact of cellular metabolism on chromatin dynamics and epigenetics. Nat Cell Biol 19(11):1298-1306. doi: 10.1038/ncb3629. PubMed PMID: 29058720; PubMed Central PMCID: PMCPMC5886854

48. Mews P, Donahue G, Drake AM, Luczak V, Abel T, Berger SL (2017) Acetyl-CoA synthetase regulates histone acetylation and hippocampal memory. Nature 546(7658):381-386. doi: 10.1038/nature22405. PubMed PMID: 28562591; PubMed Central PMCID: PMCPMC5505514

49. Ma P, Pan H, Montgomery RL, Olson EN, Schultz RM (2012) Compensatory functions of histone deacetylase 1 (HDAC1) and HDAC2 regulate transcription and apoptosis during mouse oocyte development. Proc Natl Acad Sci U S A 109(8):E481-E489. doi: 10.1073/pnas.1118403109. PubMed PMID: 22223663; PubMed Central PMCID: PMCPMC3286984

50. Ma P, Schultz RM (2013) Histone deacetylase 2 (HDAC2) regulates chromosome segregation and kinetochore function via H4K16 deacetylation during oocyte maturation in mouse. PLoS Genet 9(3):e1003377. doi: 10.1371/journal.pgen.1003377. PubMed PMID: 23516383; PubMed Central PMCID: PMCPMC3597510

51. Ma P, Schultz RM (2016) HDAC1 and HDAC2 in mouse oocytes and preimplantation embryos: Specificity versus compensation. Cell Death Differ 23(7):1119-1127. doi: 10.1038/cdd.2016.31. PubMed PMID: 27082454; PubMed Central PMCID: PMCPMC4946893

52. Wang J-J, Ge W, Zhai Q-Y, Liu J-C, Sun X-W, Liu W-X et al (2019) doi: 10.1101/803767

53. Moresi V, Carrer M, Grueter CE, Rifki OF, Shelton JM, Richardson JA et al (2012) Histone deacetylases 1 and 2 regulate autophagy flux and skeletal muscle homeostasis in mice. Proc Natl Acad Sci U S A 109(5):1649-1654. doi: 10.1073/pnas.1121159109. PubMed PMID: 22307625; PubMed Central PMCID: PMCPMC3277131

54. Cao DJ, Wang ZV, Battiprolu PK, Jiang N, Morales CR, Kong Y et al (2011) Histone deacetylase (HDAC) inhibitors attenuate cardiac hypertrophy by suppressing autophagy. Proc Natl Acad Sci U S A 108(10):4123-4128. doi: 10.1073/pnas.1015081108. PubMed PMID: 21367693; PubMed Central PMCID: PMCPMC3053983

55. Heeringa SF, Chernin G, Chaki M, Zhou W, Sloan AJ, Ji Z et al (2011) COQ6 mutations in human patients produce nephrotic syndrome with sensorineural deafness. J Clin Invest 121(5):2013-2024. doi: 10.1172/JCI45693. PubMed PMID: 21540551; PubMed Central PMCID: PMCPMC3083770

56. Peng M, Falk MJ, Haase VH, King R, Polyak E, Selak M et al (2008) Primary coenzyme Q deficiency in Pdss2 mutant mice causes isolated renal disease. PLoS Genet 4(4):e1000061. doi:

10.1371/journal.pgen.1000061. PubMed PMID: 18437205; PubMed Central PMCID: PMCPMC2291193

57. Lopez LC, Schuelke M, Quinzii CM, Kanki T, Rodenburg RJ, Naini A et al (2006) Leigh syndrome with nephropathy and CoQ10 deficiency due to decaprenyl diphosphate synthase subunit 2 (PDSS2) 
mutations. Am J Hum Genet 79(6):1125-1129. doi: 10.1086/510023. PubMed PMID: 17186472; PubMed Central PMCID: PMCPMC1698707

58. Wang Y, Song W, Wang J, Wang T, Xiong X, Qi Z et al (2020) Single-cell transcriptome analysis reveals differential nutrient absorption functions in human intestine. J Exp Med 217(2). doi: 10.1084/jem.20191130. PubMed PMID: 31753849; PubMed Central PMCID: PMCPMC7041720

59. Ge W, Wang JJ, Zhang RQ, Tan SJ, Zhang FL, Liu WX et al (2021) Dissecting the initiation of female meiosis in the mouse at single-cell resolution. Cell Mol Life Sci 78(2):695-713. doi: 10.1007/s00018020-03533-8. PubMed PMID: 32367190

60. Shimazu T, Hirschey MD, Newman J, He W, Shirakawa K, Le Moan N et al (2013) Suppression of oxidative stress by beta-hydroxybutyrate, an endogenous histone deacetylase inhibitor. Science 339(6116):211-214. doi: 10.1126/science.1227166. PubMed PMID: 23223453; PubMed Central PMCID: PMCPMC3735349

61. Shen Y, Wei W, Zhou DX (2015) Histone Acetylation Enzymes Coordinate Metabolism and Gene Expression. Trends Plant Sci 20(10):614-621. doi: 10.1016/j.tplants.2015.07.005. PubMed PMID: 26440431

62. Lakshmaiah KC, Jacob LA, Aparna S, Lokanatha D, Saldanha SC (2014) Epigenetic therapy of cancer with histone deacetylase inhibitors. J Cancer Res Ther 10(3):469-478. doi: 10.4103/09731482.137937. PubMed PMID: 25313724

63. Zhang T, He M, Zhao L, Qin S, Zhu Z, Du X et al (2021) HDAC6 regulates primordial follicle activation through mTOR signaling pathway. Cell Death Dis 12(6):559. doi: 10.1038/s41419-021-03842-1. PubMed PMID: 34052832; PubMed Central PMCID: PMCPMC8164630

64. Miao Y, Cui Z, Gao Q, Rui R, Xiong B (2020) Nicotinamide Mononucleotide Supplementation Reverses the Declining Quality of Maternally Aged Oocytes. Cell Reports 32(5):107987. doi: 10.1016/j.celrep.2020.107987

65. Zhang H, Pan Z, Ju J, Xing C, Li X, Shan M et al (2020) DRP1 deficiency induces mitochondrial dysfunction and oxidative stress-mediated apoptosis during porcine oocyte maturation. J Anim Sci Biotechnol 11(1):77. doi: 10.1186/s40104-020-00489-4

66. Babayev E, Seli E (2015) Oocyte mitochondrial function and reproduction. Curr Opin Obstet Gynecol 27(3):175-181. doi:

10.1097

/GC0.0000000000000164. PubMed PMID: 25719756; PubMed Central PMCID: PMCPMC4590773

67. Luo D, Zhang JB, Li SP, Liu W, Yao XR, Guo H et al (2020) Imperatorin Ameliorates the AgingAssociated Porcine Oocyte Meiotic Spindle Defects by Reducing Oxidative Stress and Protecting Mitochondrial Function. Front Cell Dev Biol 8:592433 PubMed PMID: 33409275; PubMed Central PMCID: PMCPMC7779485

68. Wu LL-Y, Dunning KR, Yang X, Russell DL, Lane M, Norman RJ et al (2010) High-Fat Diet Causes Lipotoxicity Responses in Cumulus-Oocyte Complexes and Decreased Fertilization Rates. Endocrinology 151(11):5438-5445. doi: 10.1210/en.2010-0551 
69. May-Panloup P, Boucret L, Chao de la Barca J-M, Desquiret-Dumas V, Ferré-L'Hotellier V, Morinière C et al (2016) Ovarian ageing: the role of mitochondria in oocytes and follicles. Hum Reprod Update 22(6):725-743. doi: 10.1093/humupd/dmw028

70. Chappel S (2013) The role of mitochondria from mature oocyte to viable blastocyst. Obstet Gynecol Int 2013:183024. doi: 10.1155/2013/183024. PubMed PMID: 23766762; PubMed Central PMCID: PMCPMC3671549

71. Yang Q, Cong L, Wang Y, Luo X, Li H, Wang H et al (2020) Increasing ovarian NAD+ levels improve mitochondrial functions and reverse ovarian aging. Free Radic Biol Med 156:1-10. doi: 10.1016/j.freeradbiomed.2020.05.003

72. Rodríguez-Varela C, Labarta E (2020) Clinical Application of Antioxidants to Improve Human Oocyte Mitochondrial Function: A Review. Antioxidants 9(12):1197. doi: 10.3390/antiox9121197

\section{Figures}




\section{Figure 1}
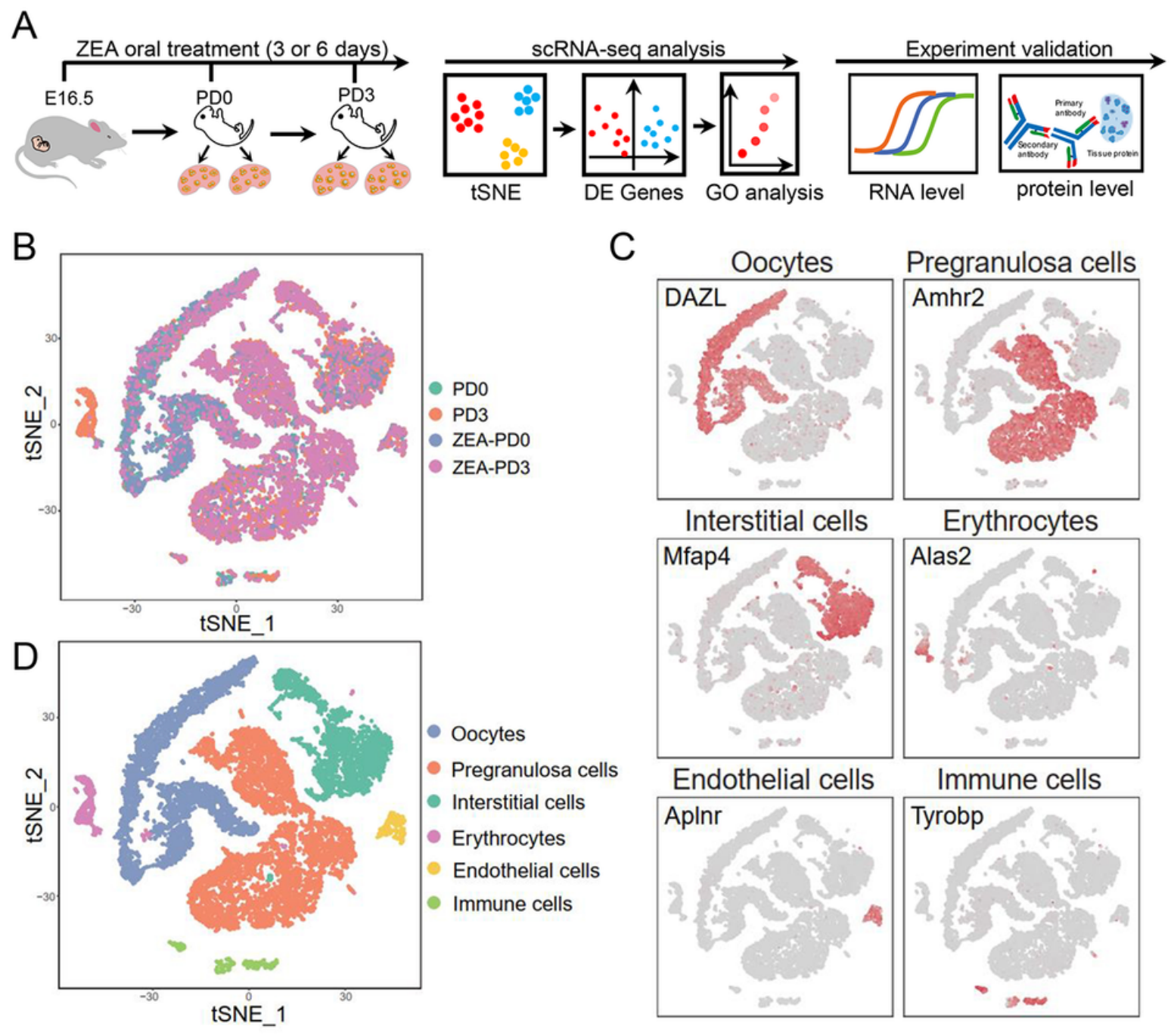

\section{Figure 1}

\section{Experimental design and identification of ovarian cell types using scRNA-seq.}

(A) The overall experimental framework of the study, including mouse handling, single cell data analysis, and experimental validation. (B) tSNE plot of ovarian cells from the four experimental groups. (C) Feature plots of cell classic markers gene to identify the ovarian cell types. (D) Six ovarian cell types identified by tSNE plot 
Figure 2

A

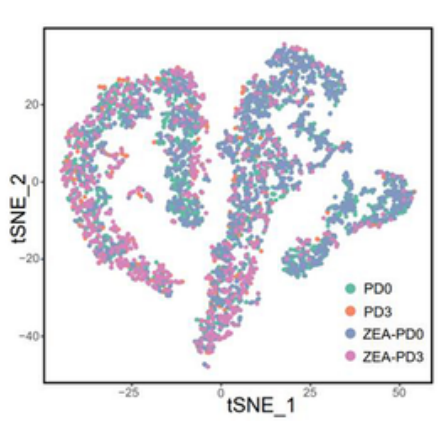

B

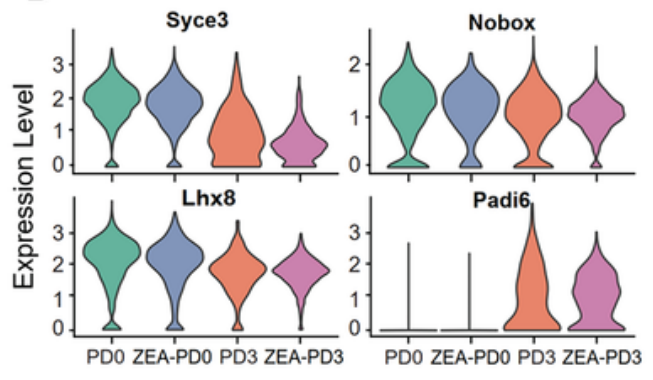

C

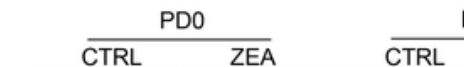
PD3
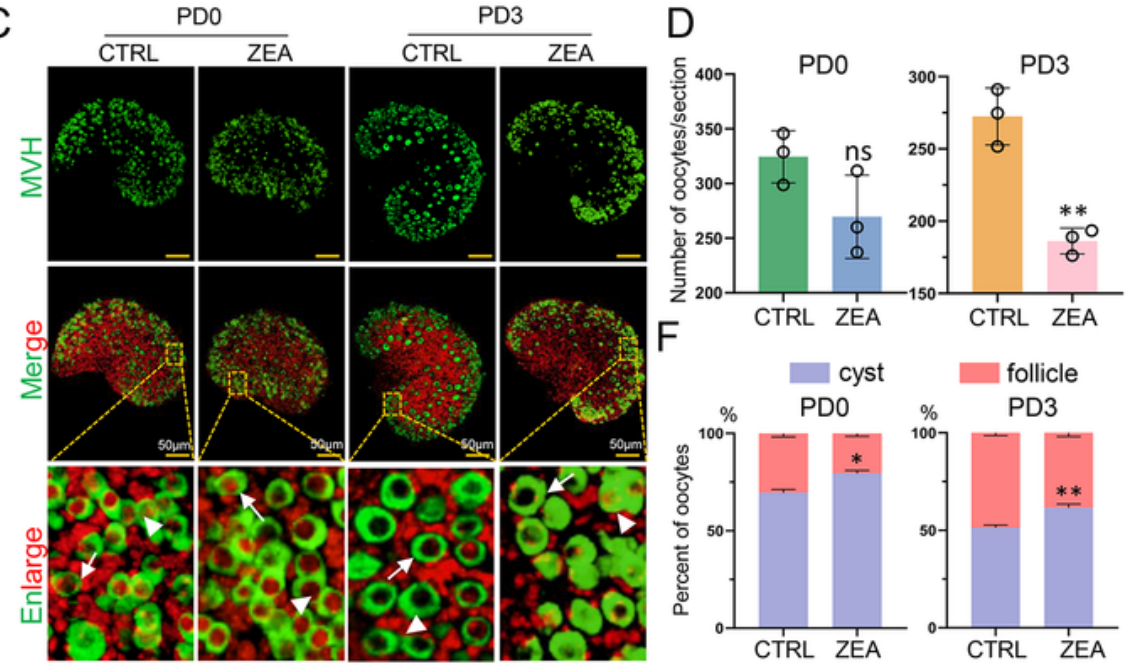

$\mathrm{E}$
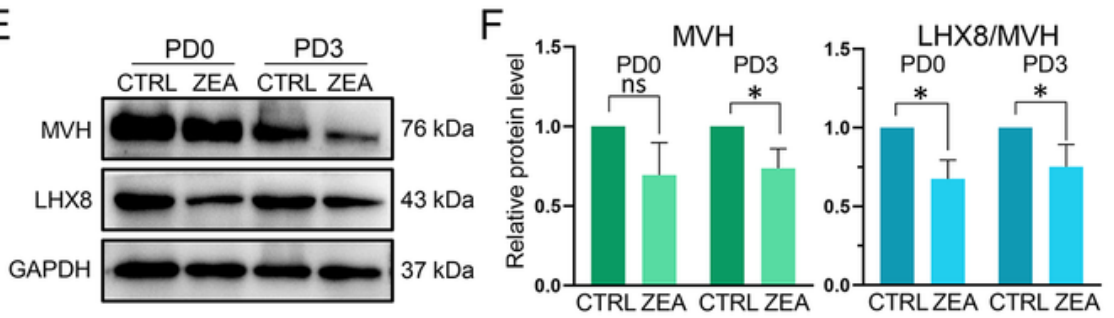

Figure 2

Maternal ZEA exposure impairs primordial follicles formation in offspring.

(A) tSNE plot of oocytes based on four sample sets. (B) Violin plots showing the average expression level in the four experimental groups of classic oocyte genes (Syce3, Lhx8, Nobox, Padi6). (C) Representative IF for MVH (green) in ovaries of the four experimental groups. Arrowheads, cysts, arrows, primordial 
follicles. Scale bar $=50 \mu \mathrm{m}$. (D) Number of MVH positive oocytes (upper) and their percentage in cysts or PFs (below) in the four experimental groups, calculated as the mean of counts in eight sections per ovary. For each group at least three ovaries were analyzed. Data are shown as mean $\pm \mathrm{SD}\left({ }^{\star} P<0.05,{ }^{\star \star} P<\right.$ 0.01). (E-F) Representative WB for MVH and LHX8 proteins and quantitative evaluation of their expression in the four experimental groups (the levels of LHX8 and MVH were calculated relatively to $\mathrm{MVH}$ and GAPDH, respectively). All analyses were repeated at least three times on different samples. Data are shown as mean \pm SD $\left({ }^{\star} P<0.05, * \star P<0.01\right)$

\section{Figure 3}

A ZEA vs. CTRL
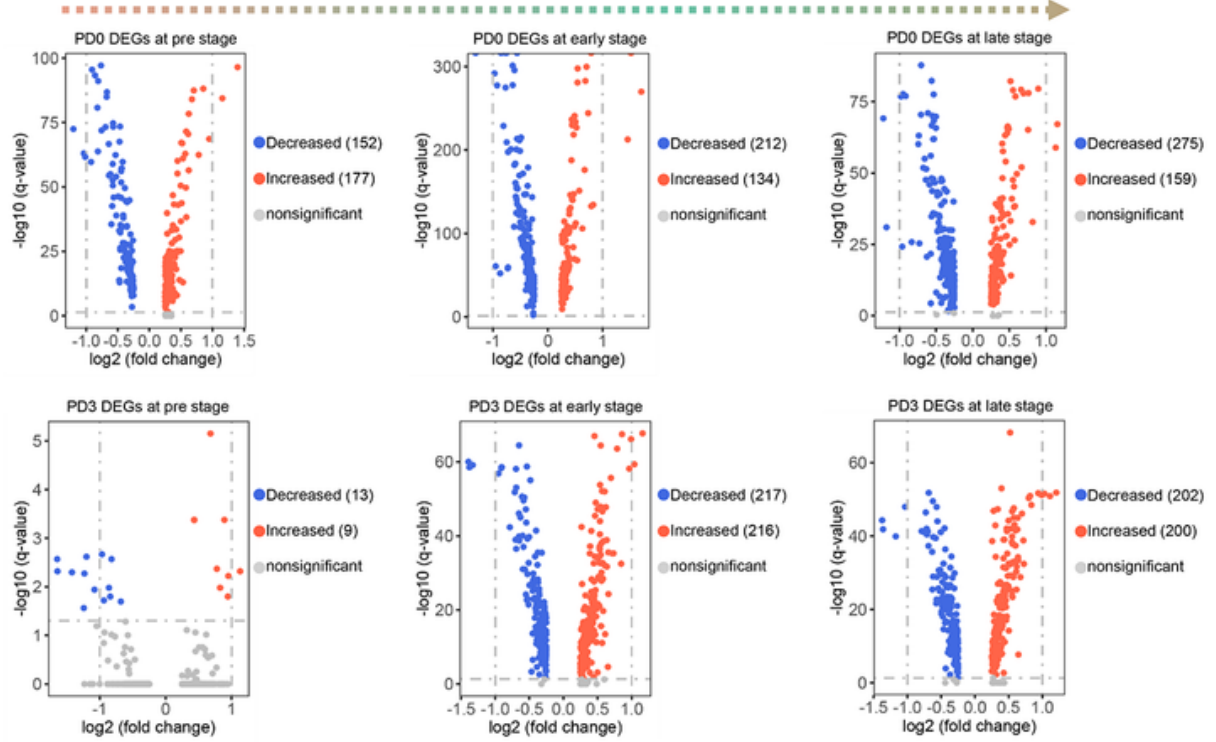

\section{B Pre-PD0 Pre-PD3}
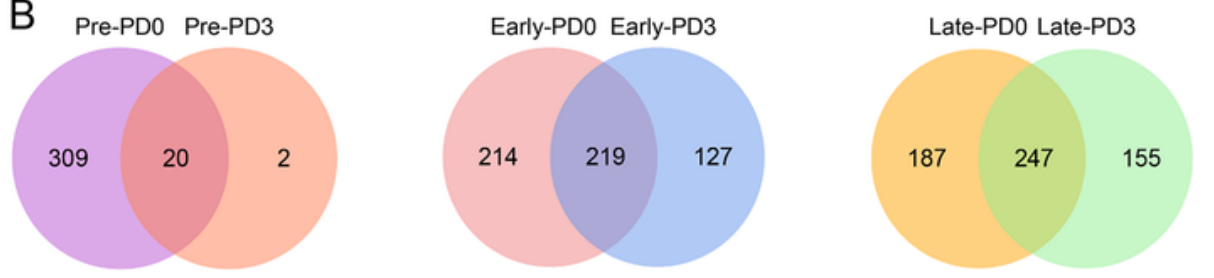

C

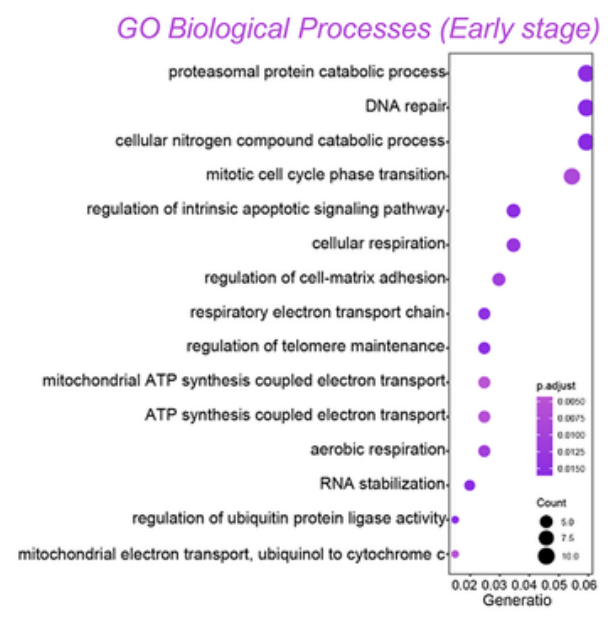

GO Biological Processes (Late stage)

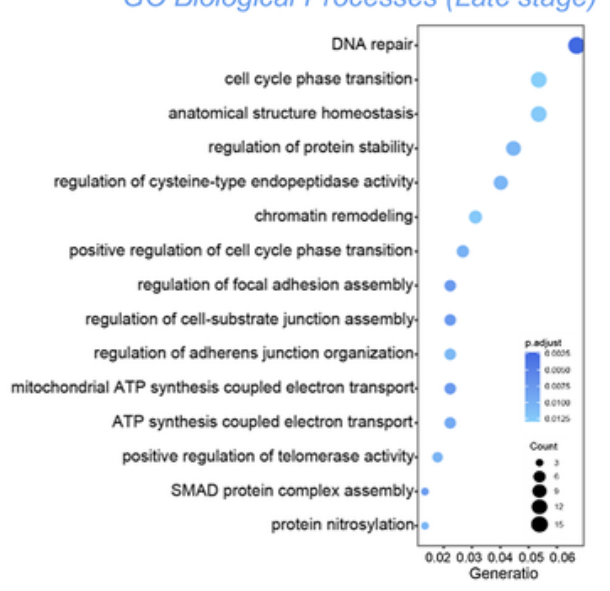


Figure 3

Differential gene expression analysis in oocytes of offspring ovaries from ZEA exposed mothers.

(A) Volcano plots of differentially expressed genes (DEGs, down-regulated, blue, up-regulated, red) of oocytes from PD0 and PD3 ovaries at the indicated PF stages in ZEA exposed mothers in comparison to CTRL. (B) Venn plots of DEGs in oocytes at the three PF stages. (C) GO enrichment analysis of DEGs in oocytes at early- and late- PF stages 


\section{Figure 4}

A
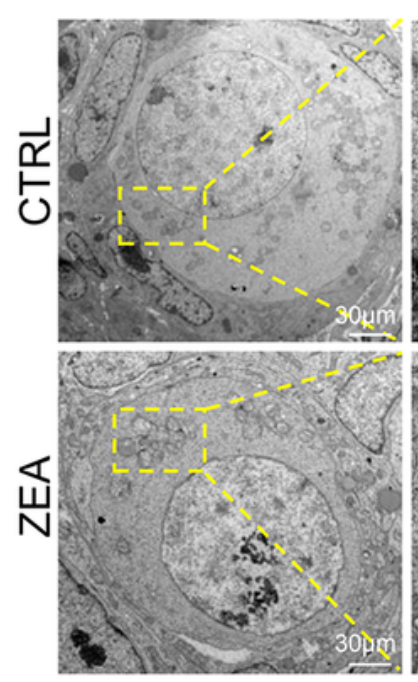

oocyte
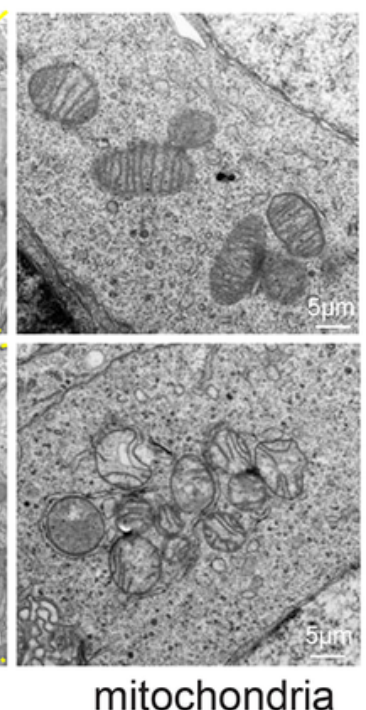

B
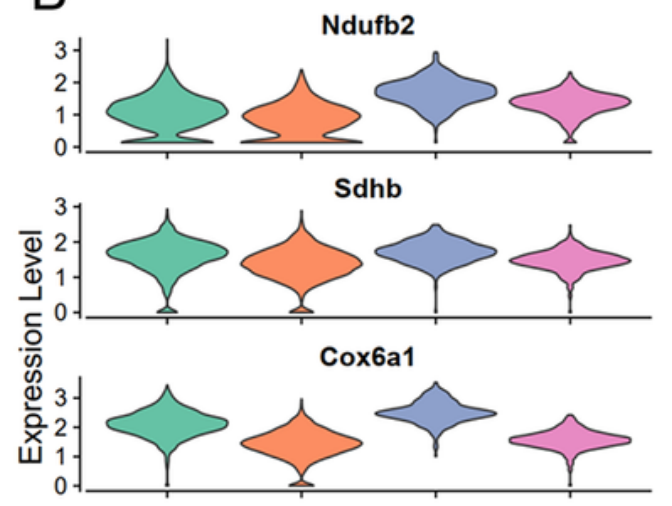

Atp5I

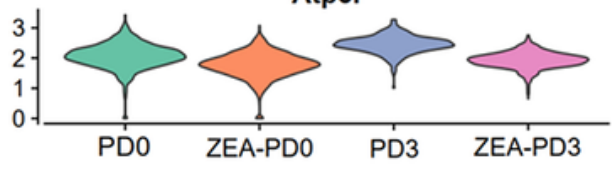

C
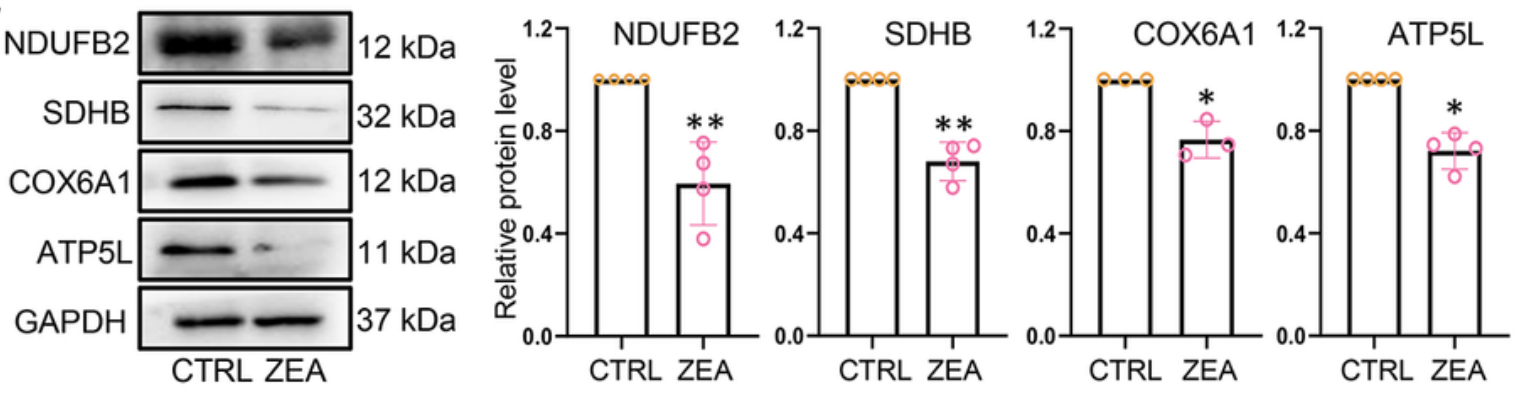

D
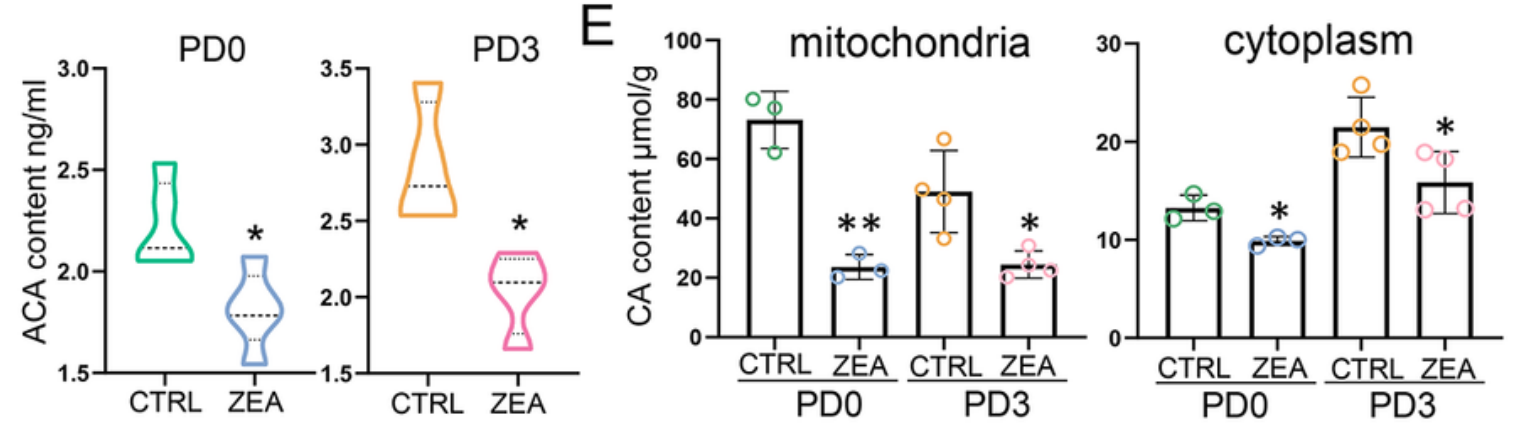

Figure 4

\section{Effects of ZEA on mitochondria morphology and activities.}

(A) TEM images of mitochondrial morphologies in CTRL and ZEA exposed oocytes. Note, CTRL group: mitochondria are intact ellipse with many inner cristae, ZEA group: mitochondria become fragmentation and vacuolization, Scale bar $=30 \mu \mathrm{m}$ and $5 \mu \mathrm{m}$. Fifteen sections from three ovaries were examined in 
each group. (B) Violin plots showing the expression level of the transcripts of phosphorylation complex proteins (Ndufb2, Sdhb, Cox6a 1, Atp5l) in the ovaries of the four experimental groups. (C) Representative WB of the same proteins. (D) Amount of ACA of offspring ovaries in CTRL and ZEA exposed group. (E) Mitochondria and cytoplasm content of CA in CTRL and ZEA exposed offspring ovaries. All analyses were repeated at least three times on different samples. Data are shown as mean $\pm \operatorname{SD}\left({ }^{\star} P<0.05, \star \star P<\right.$ $0.01)$

Figure 5
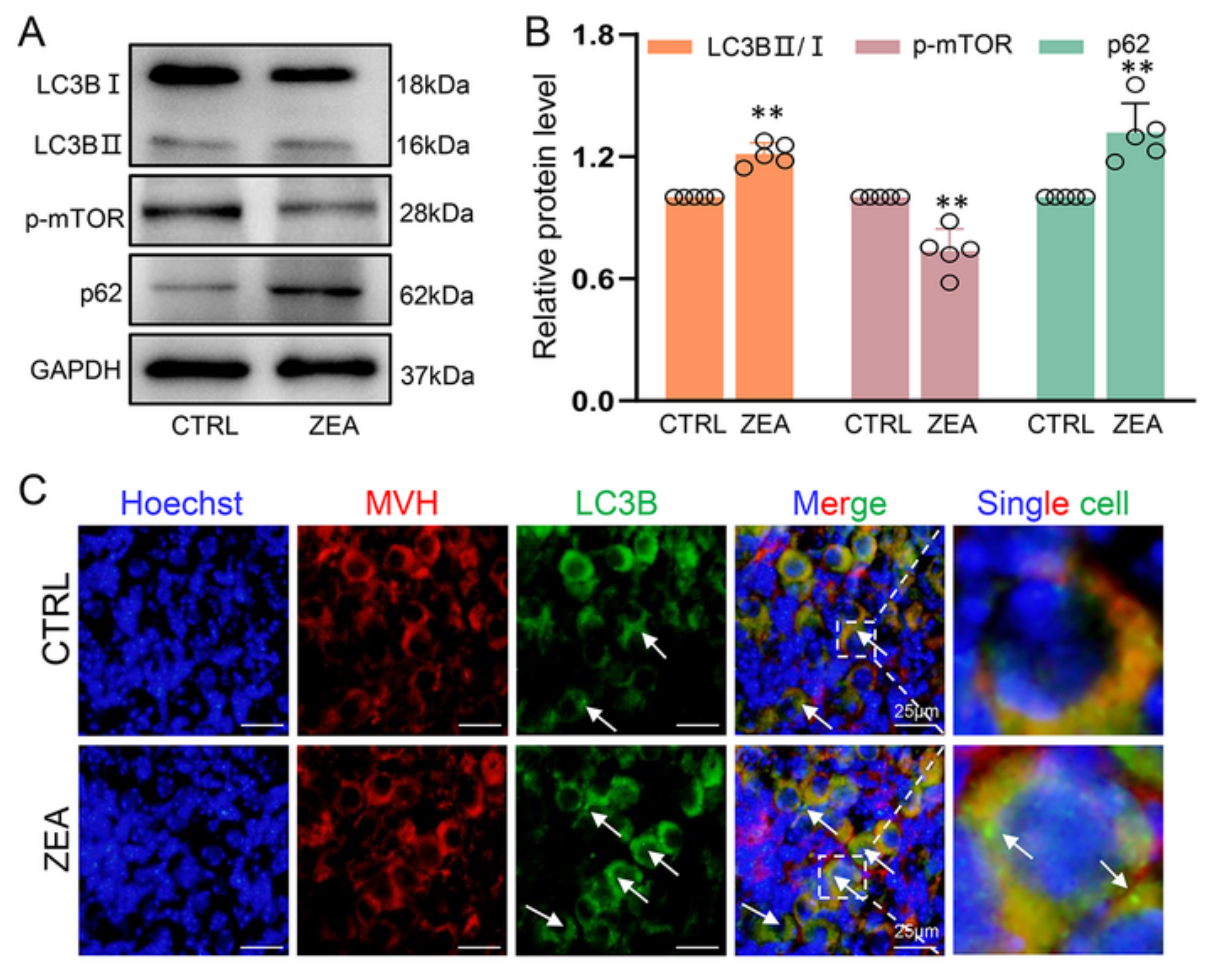

D

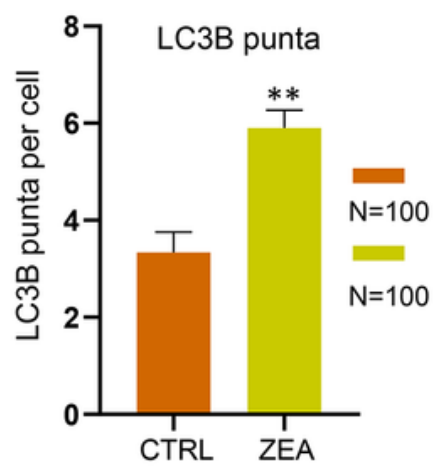

E

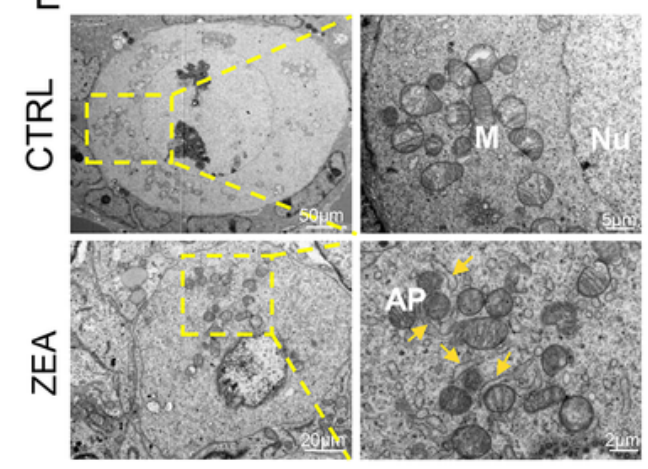


Figure 5

\section{ZEA induces autophagy in oocytes of PD3 offspring ovaries.}

(A-B) Representative WB and quantification of the expression of the autophagic proteins LC3B II/I, p62 and $p-m T O R$ in PD3 offspring ovaries from CTRL and ZEA exposed mothers. For each group at least thirty ovaries were used. Data are shown as mean \pm SD $\left({ }^{\star} P<0.05\right.$, $\left.{ }^{\star *} P<0.01\right)$. (C-D) IF images and the quantities of LC3B (green) in the cytoplasm of MVH (red) positive oocytes, arrows: LC3B puncta structures (the punctate structure reflects the degree of autophagy). scale bar $=25 \mu \mathrm{m}$. Nine sections from three ovaries were examined in each group. (E) TEM observations of phagophore and autophagosomes (arrows) in the CTRL and ZEA exposed oocytes. AP, autophagosome, $M$, mitochondria, $\mathrm{Nu}$, oocyte nucleus. Scale bar $=50 \mu \mathrm{m}$ and $5 \mu \mathrm{m}$. Fifteen sections from three ovaries were examined. All analyses were repeated at least three times. On different samples Data are shown as mean \pm SD $\left({ }^{\star} P<\right.$ $0.05, * \star P<0.01)$ 
Figure 6
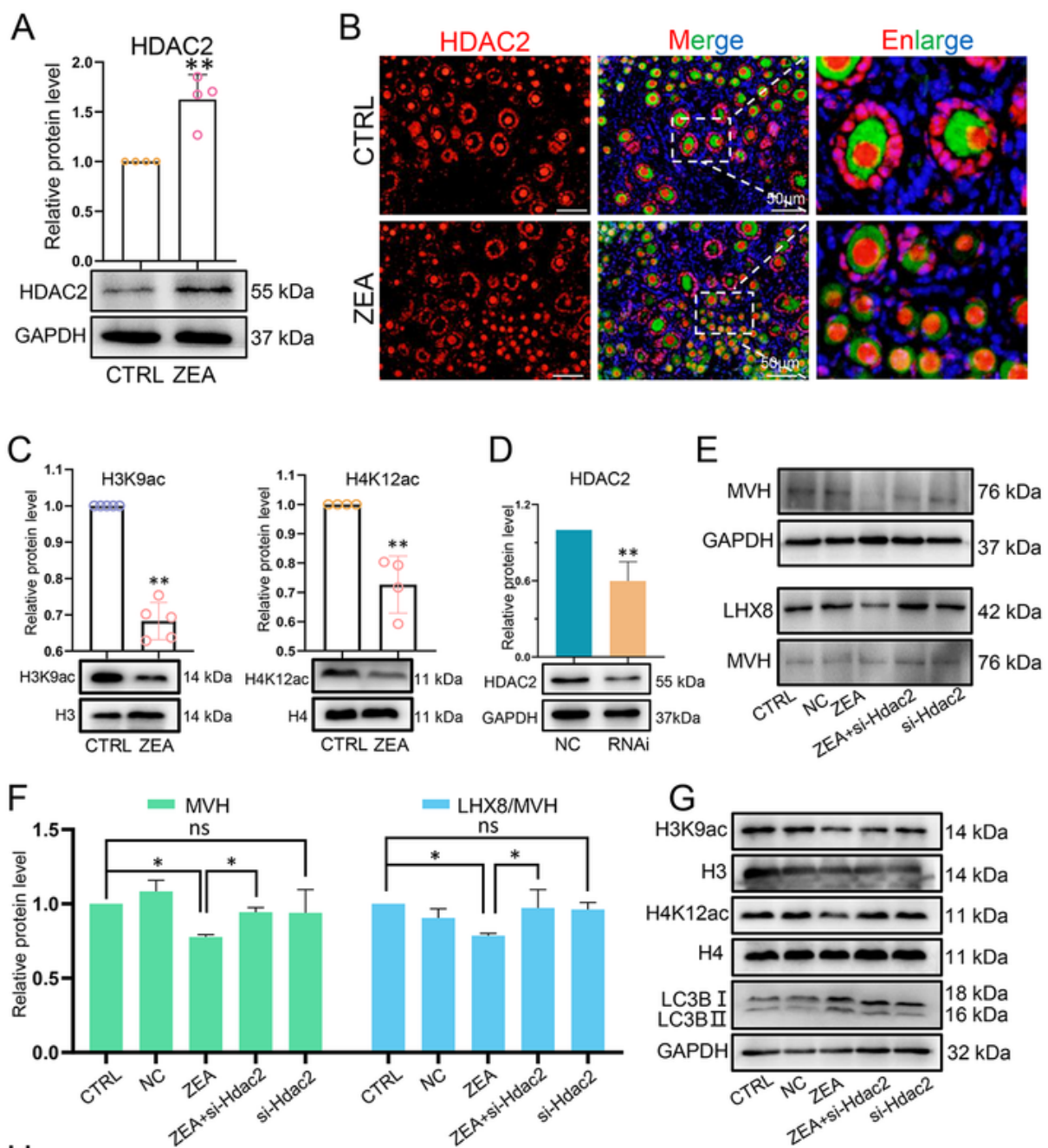

G
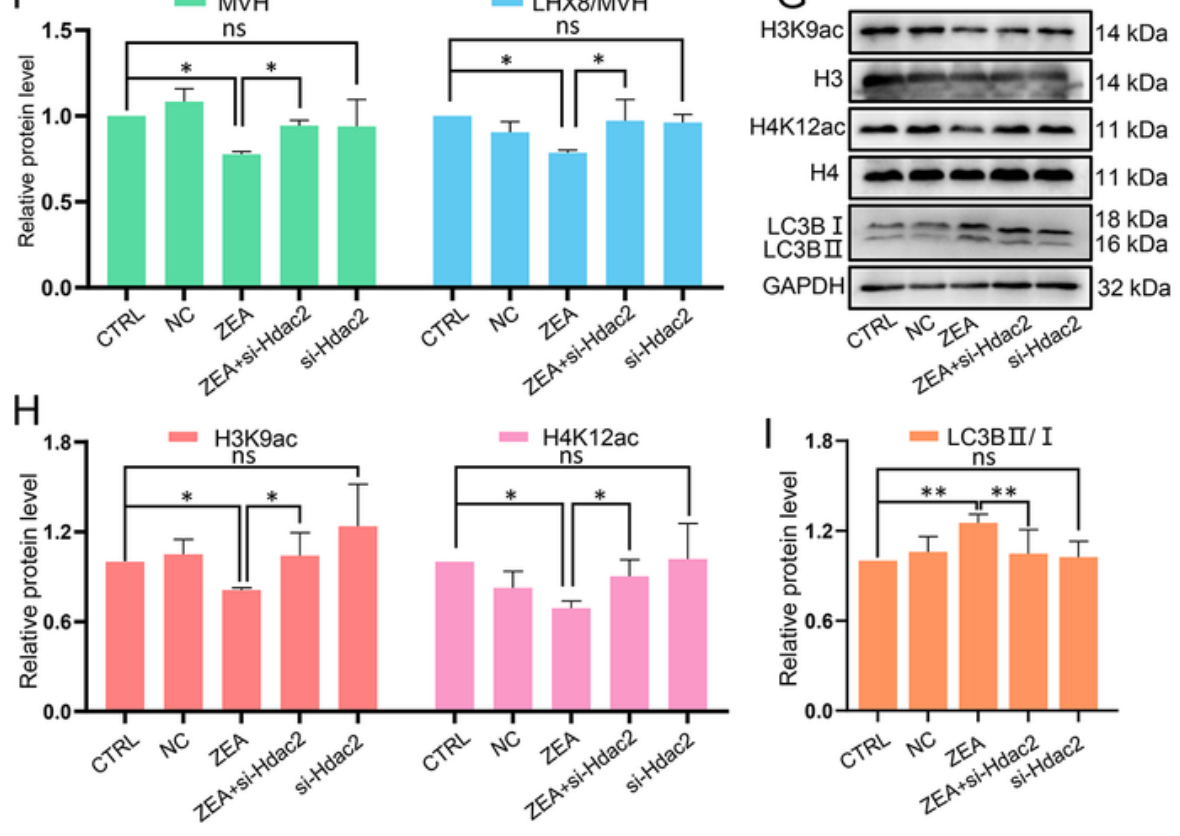

\section{Figure 6}

\section{ZEA increases HDAC2 levels and alters histone acetylation in the offspring ovaries.}

(A) Representative WB for HDAC2 in PD3 offspring ovaries from CTRL and ZEA exposed mothers. (B) IF for HDAC2 in the same ovaries as above. Scale bar $=50 \mu \mathrm{m}$. Twenty sections from three ovaries were examined in each group. (C) WB for H3K9ac and H4K12ac in offspring ovaries after ZEA exposed 
mothers. (D) WB for HDAC2 in newborn PD0 ovaries cultured for three days with and without si-Hdac2 treatment. (E-F) WB for MVH and LHX8 in newborn PD0 ovaries cultured for three days under the indicated conditions (the level of LHX8 was calculated relatively to MVH, that of MVH to GAPDH). (G-H) WB for H3K9ac and H4K12ac in newborn PD0 ovaries cultured for three days under the indicated conditions. (I) WB and quantitative evaluation of the LC3B II/ $₫$ expression level in newborn ovaries cultured for three days under the indicated conditions, note that in the presence of si-Hdac2 the effects of ZEA on the expression of these proteins were nearly abolished. All analyses were repeated at least three times. Data are shown as mean $\pm \mathrm{SD}\left({ }^{*} P<0.05,{ }^{*} P<0.01\right)$. NC (negative control) are ovaries cultured in the presence of the drug solvent only 
Figure 7

A

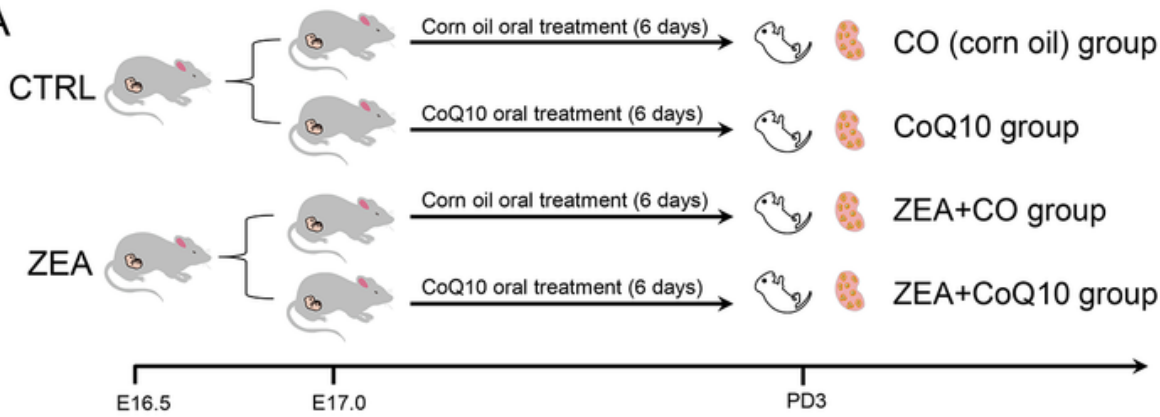

B

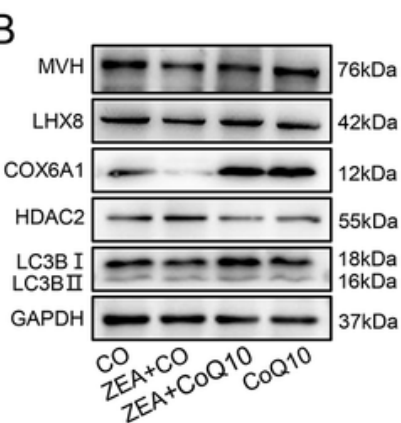

C
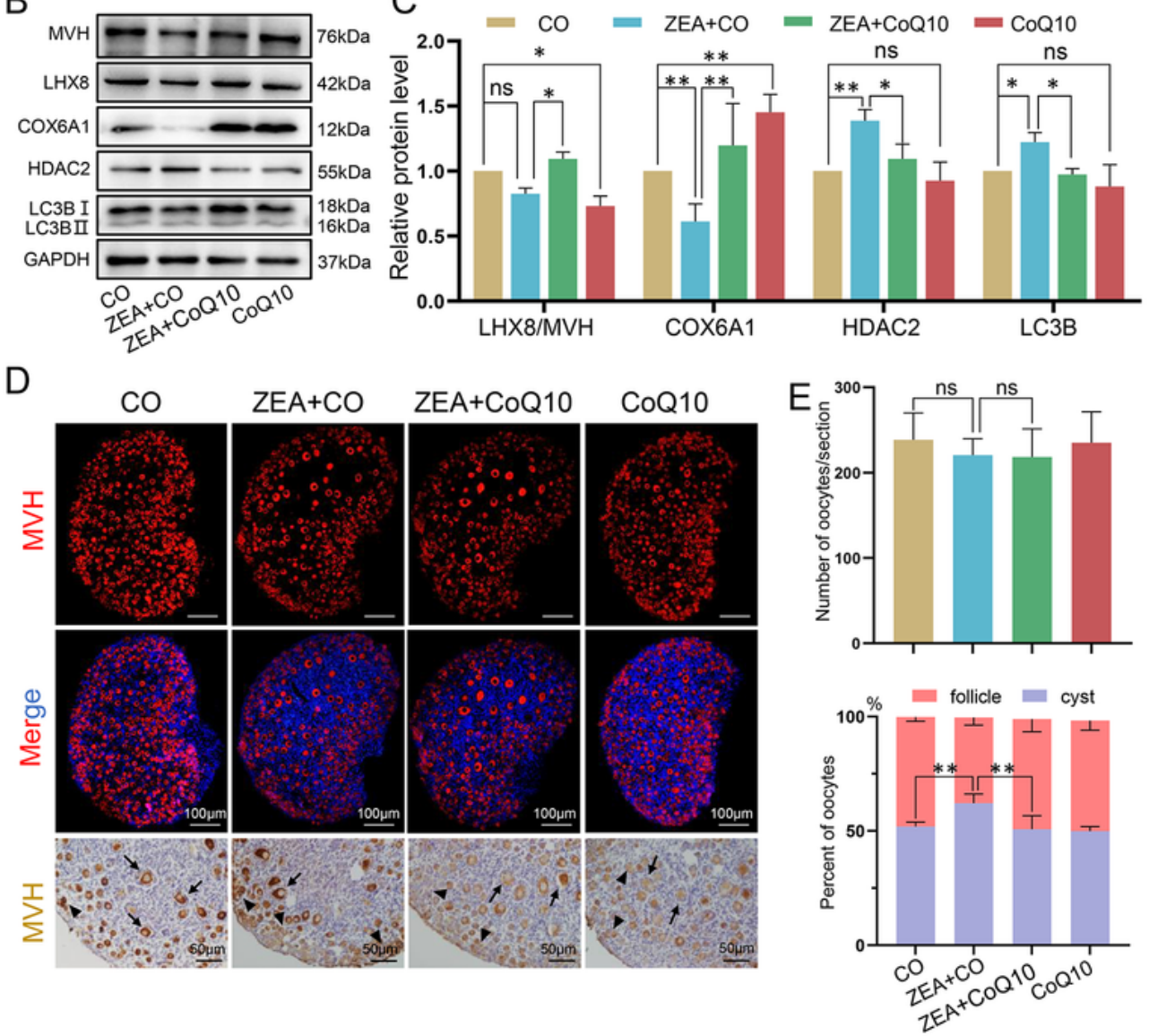

Figure 7

CoQ10 counteracts the effect of ZEA on the offspring ovaries.

(A) A timeline and grouping diagram of CoQ10 administration (to eliminate the influence of CoQ10 solvent on the experimental results, CTRL group and ZEA group were also orally given CoQ10 solvent). (BC) Representative WB for MVH, LHX8, HDAC2, COX6A1 and LC3B and quantification of their expression in 
protein extracts of PD3 ovaries of offspring from mothers treated as indicated. Note that whereas ZEA altered the expression levels of these proteins, CoQ10 administered with the drug counteracted these effects. (D) Representative IF (upper) and IHC (lower) for MVH of sections of ovaries of PD3 newborns from mothers treated with the indicated compounds. Arrowheads, cysts, arrows, primordial follicles. (E) The number and percentage of oocytes in follicles towards in cysts in the same groups. Note that while the numbers of the MVH positive oocytes were similar in all groups, ZEA reduced those in follicles and CoQ10 reverted the percentage to control values. Scale bar $=100 \mu \mathrm{m}$ and $50 \mu \mathrm{m}$, respectively. Forty sections from five ovaries in each group were examined 


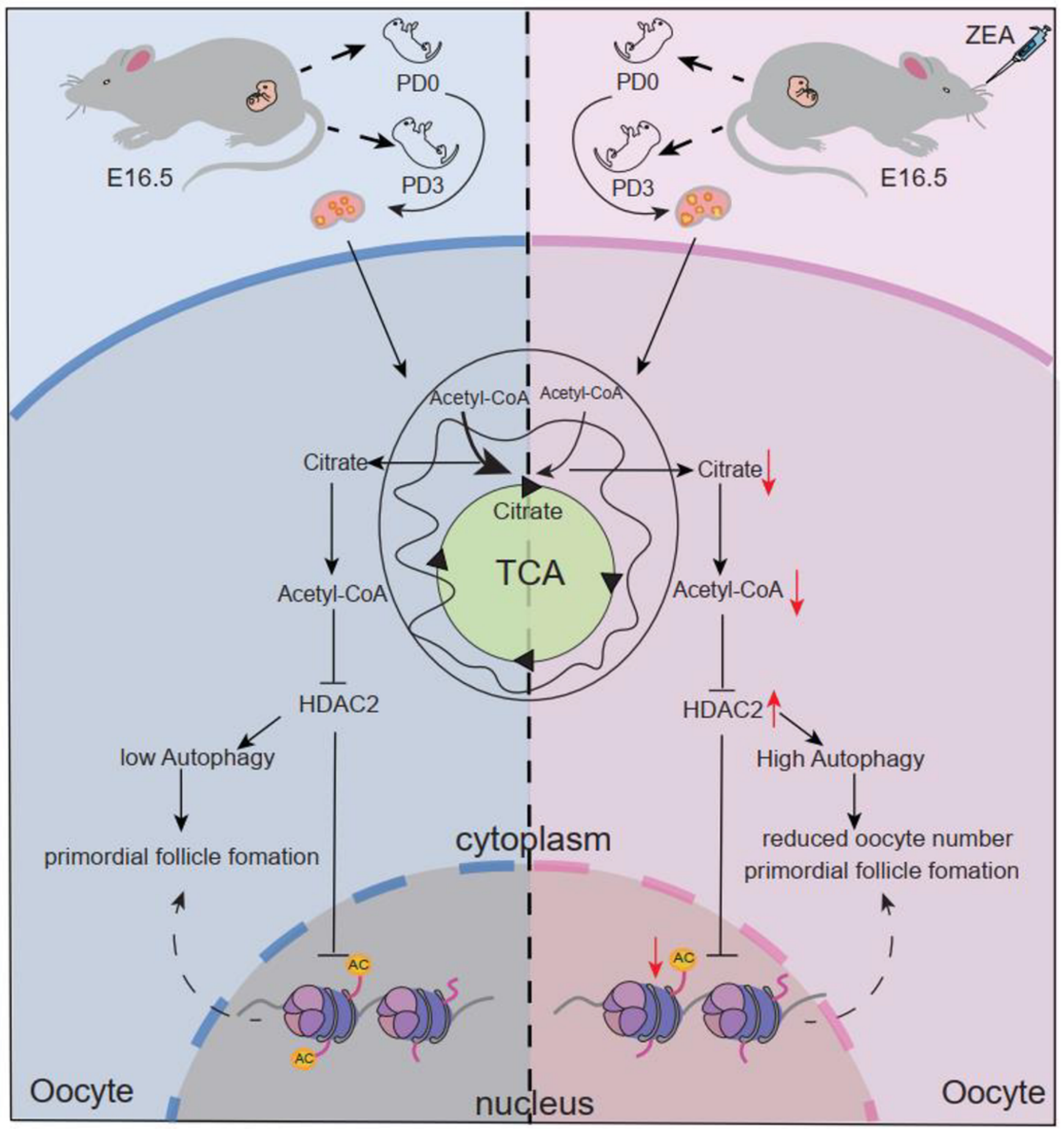

\section{Figure 8}

A sketch depicting the experimental design and the main molecular pathways affected by ZEA in oocytes identified in the present paper.

Briefly, decreased production of Acetyl-CoA and Citrate, as a consequence of ZEA action on the oocyte mitochondria, leads to enhanced expression/activity of HDAC2 that alters the expression of genes 
regulating autophagy and PF assembly through modifying histone acetylation

\section{Supplementary Files}

This is a list of supplementary files associated with this preprint. Click to download.

- FigS1.jpg

- FigS2.jpg

- FigS3.jpg

- FigS4.jpg

- TableS1Listofprimers.doc

- TableS2Listofprimaryantibodies.docx

- TableS3samplepreprocessingparameters.docx

- TableS4Top50markergenesofcellclustersinovaries.csv

- TableS5DEGsofZENexposureatPD0andPD3.csv

- TableS6DEGsofZENexposureat3stage.csv 